University of Wollongong

Research Online

Australian Institute for Innovative Materials -

Papers

Australian Institute for Innovative Materials

$1-1-2014$

Electrostrictive effect in ferroelectrics: An alternative approach to improve piezoelectricity

Fei Li

Xi'an Jiaotong University, lifei1216@gmail.com

Li Jin

Xi'an Jiaotong University

Zhuo Xu

Xi'an Jiaotong University

Shujun Zhang

The Pennsylvania State University, shujun@uow.edu.au

Follow this and additional works at: https://ro.uow.edu.au/aiimpapers

Part of the Engineering Commons, and the Physical Sciences and Mathematics Commons

Research Online is the open access institutional repository for the University of Wollongong. For further information contact the UOW Library: research-pubs@uow.edu.au 


\title{
Electrostrictive effect in ferroelectrics: An alternative approach to improve piezoelectricity
}

\begin{abstract}
Electrostriction plays an important role in the electromechanical behavior of ferroelectrics and describes a phenomenon in dielectrics where the strain varies proportional to the square of the electric field/ polarization. Perovskite ferroelectrics demonstrating high piezoelectric performance, including BaTiO3, $\mathrm{Pb}(\mathrm{Zr1-xTix}) 03$, and relaxor-PbTiO3 materials, have been widely used in various electromechanical devices. To improve the piezoelectric activity of these materials, efforts have been focused on the ferroelectric phase transition regions, including shift the composition to the morphotropic phase boundary or shift polymorphic phase transition to room temperature. However, there is not much room left to further enhance the piezoelectric response in perovskite solid solutions using this approach. With the purpose of exploring alternative approaches, the electrostrictive effect is systematically surveyed in this paper. Initially, the techniques for measuring the electrostrictive effect are given and compared. Second, the origin of electrostriction is discussed. Then, the relationship between the electrostriction and the microstructure and macroscopic properties is surveyed. The electrostrictive properties of ferroelectric materials are investigated with respect to temperature, composition, phase, and orientation. The relationship between electrostriction and piezoelectric activity is discussed in detail for perovskite ferroelectrics to achieve new possibilities for piezoelectric enhancement. Finally, future perspectives for electrostriction studies are proposed. 2014 AIP Publishing LLC.
\end{abstract}

\section{Keywords}

improve, piezoelectricity, approach, electrostrictive, alternative, effect, ferroelectrics

\author{
Disciplines \\ Engineering | Physical Sciences and Mathematics
}

\section{Publication Details}

Li, F., Jin, L., Xu, Z. \& Zhang, S. (2014). Electrostrictive effect in ferroelectrics: An alternative approach to improve piezoelectricity. Applied Physics Reviews, 1 (1), 011103-1-011103-21. 


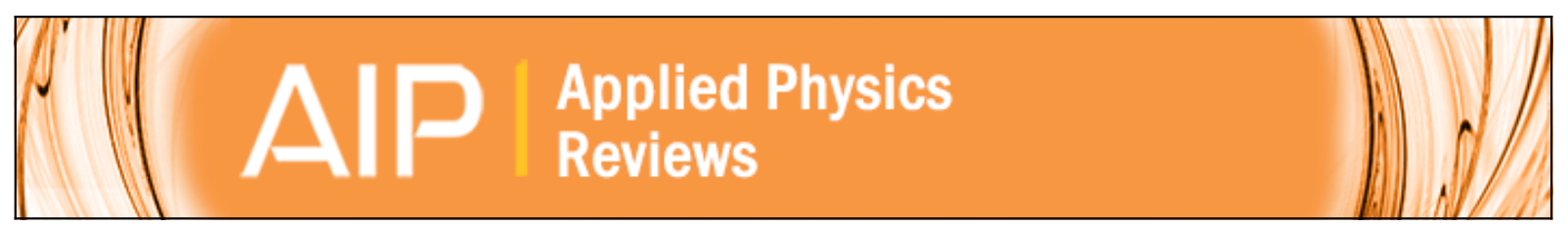

\section{Electrostrictive effect in ferroelectrics: An alternative approach to improve piezoelectricity}

Fei Li, Li Jin, Zhuo Xu, and Shujun Zhang

Citation: Applied Physics Reviews 1, 011103 (2014); doi: 10.1063/1.4861260

View online: http://dx.doi.org/10.1063/1.4861260

View Table of Contents: http://scitation.aip.org/content/aip/journal/apr2/1/1?ver=pdfcov

Published by the AIP Publishing

\section{Articles you may be interested in}

Phase transition behavior and defect chemistry of [001]-oriented $0.15 \mathrm{~Pb}(\ln 1 / 2 \mathrm{Nb} 1 / 2) \mathrm{O} 3-0.57 \mathrm{~Pb}(\mathrm{Mg} 1 / 3 \mathrm{Nb} 2 / 3) \mathrm{O} 3-$ $0.28 \mathrm{PbTiO}-\mathrm{Mn}$ single crystals

J. Appl. Phys. 117, 244102 (2015); 10.1063/1.4922790

Enhanced dielectric, ferroelectric, and electrostrictive properties of $\mathrm{Pb}(\mathrm{Mg} 1 / 3 \mathrm{Nb} 2 / 3) 0.9 \mathrm{Ti} 0.103$ ceramics by $\mathrm{ZnO}$ modification

J. Appl. Phys. 113, 204101 (2013); 10.1063/1.4801881

Effective piezoelectric response of twin walls in ferroelectrics

J. Appl. Phys. 113, 187222 (2013); 10.1063/1.4801988

Structure, piezoelectric, and ferroelectric properties of $\mathrm{BaZrO} 3$ substituted $\mathrm{Bi}(\mathrm{Mg} 1 / 2 \mathrm{Ti} 1 / 2) \mathrm{O} 3-\mathrm{PbTiO} 3$ perovskite J. Appl. Phys. 111, 104118 (2012); 10.1063/1.4722286

Mechanisms of electrostriction and giant piezoelectric effect in relaxor ferroelectrics

J. Appl. Phys. 93, 9947 (2003); 10.1063/1.1575915

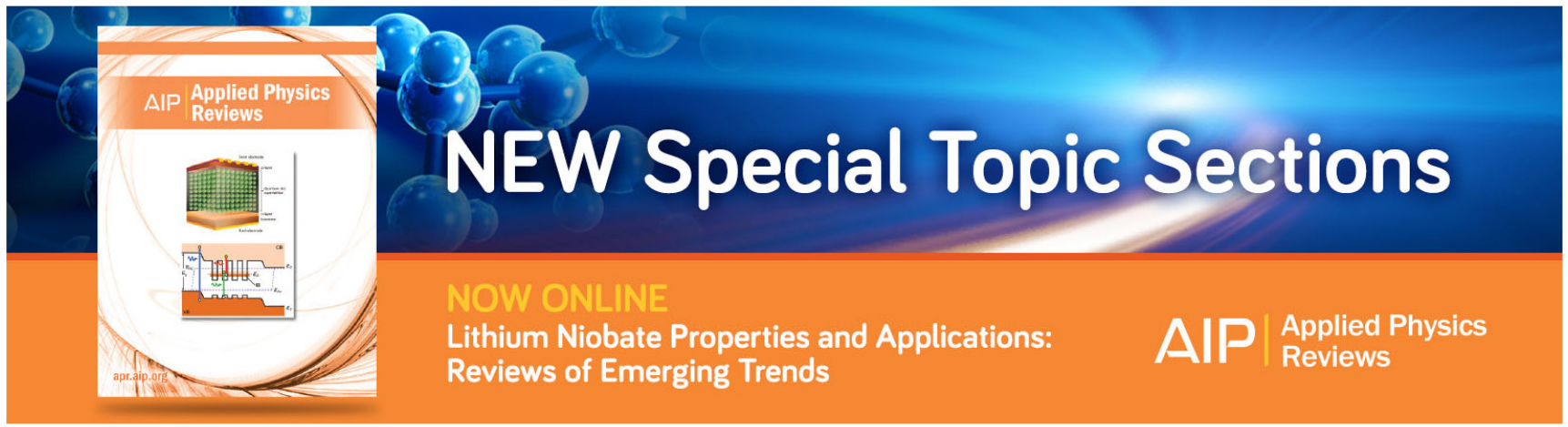




\title{
Electrostrictive effect in ferroelectrics: An alternative approach to improve piezoelectricity
}

\author{
Fei Li, ${ }^{1}$ Li Jin, ${ }^{1}$ Zhuo Xu, ${ }^{1}$ and Shujun Zhang ${ }^{2, a)}$ \\ ${ }^{1}$ Electronic Materials Research Laboratory, Key Laboratory of the Ministry of Education and International \\ Center for Dielectric Research, Xi' an Jiaotong University, Xi' an 710049, China \\ ${ }^{2}$ Materials Research Institute, Pennsylvania State University, University Park, Pennsylvania 16802, USA
}

(Received 14 July 2013; accepted 2 October 2013; published online 15 January 2014)

\begin{abstract}
Electrostriction plays an important role in the electromechanical behavior of ferroelectrics and describes a phenomenon in dielectrics where the strain varies proportional to the square of the electric field/polarization. Perovskite ferroelectrics demonstrating high piezoelectric performance, including $\mathrm{BaTiO}_{3}, \mathrm{~Pb}\left(\mathrm{Zr}_{1-x} \mathrm{Ti}_{x}\right) \mathrm{O}_{3}$, and relaxor- $\mathrm{PbTiO}_{3}$ materials, have been widely used in various electromechanical devices. To improve the piezoelectric activity of these materials, efforts have been focused on the ferroelectric phase transition regions, including shift the composition to the morphotropic phase boundary or shift polymorphic phase transition to room temperature. However, there is not much room left to further enhance the piezoelectric response in perovskite solid solutions using this approach. With the purpose of exploring alternative approaches, the electrostrictive effect is systematically surveyed in this paper. Initially, the techniques for measuring the electrostrictive effect are given and compared. Second, the origin of electrostriction is discussed. Then, the relationship between the electrostriction and the microstructure and macroscopic properties is surveyed. The electrostrictive properties of ferroelectric materials are investigated with respect to temperature, composition, phase, and orientation. The relationship between electrostriction and piezoelectric activity is discussed in detail for perovskite ferroelectrics to achieve new possibilities for piezoelectric enhancement. Finally, future perspectives for electrostriction studies are proposed. (C) 2014 AIP Publishing LLC. [http://dx.doi.org/10.1063/1.4861260]
\end{abstract}

\section{TABLE OF CONTENTS}

I. INTRODUCTION

II. THE DETERMINATION OF THE

ELECTROSTRICTIVE COEFFICIENTS. .......

A. Electrostrictive coefficients measured by strain versus the polarization/electric field . .

B. Electrostrictive coefficients measured using the dielectric permittivity versus the applied stress ..........................

C. Electrostrictive coefficients determined using the piezoelectric coefficients........

D. Electrostrictive coefficients determined from the lattice parameters .............

E. Electrostrictive coefficients determined from the dielectric permittivity under a DC-

biased electric field .................

III. THE ORIGIN OF ELECTROSTRICTION......

IV. ELECTROSTRICTION WITH RESPECT TO THE MICROSCOPIC AND MACROSCOPIC CHARACTERISTICS $\ldots \ldots \ldots \ldots \ldots \ldots \ldots$

\footnotetext{
${ }^{\text {a) }}$ Author to whom correspondence should be addressed. Electronic mail: soz1@psu.edu; shujunzhang@gmail.com.
}

A. Microscopic characteristics versus the electrostrictive effect................

B. Macroscopic characteristics versus the electrostrictive effect.................

1. Dielectric and elastic responses versus the electrostrictive effect............

2. Thermal expansion versus the electrostrictive effect ..............

\section{ELECTROSTRICTION IN PEROVSKITE} FERROELECTRICS ...................

A. Electrostrictive effect versus ferroelectric phase transitions ................... 1. Polymorphic phase transition (PPT, phase transitions induced by temperature) $\ldots \ldots \ldots \ldots \ldots \ldots \ldots \ldots$

2. Morphotropic phase boundary (MPB, phase transitions induced by composition) .............. 13

B. Orientation dependence of electrostriction .. 14

C. Electrostrictive coefficient $Q$ versus the electromechanical properties ............. 1. Electrostrictive coefficient $Q$ versus the electric field-induced strain $\ldots \ldots \ldots \ldots$

2. Electrostrictive coefficient $Q$ versus piezoelectric activity $\ldots \ldots \ldots \ldots \ldots \ldots$ 
3. Can piezoelectric activity be improved with electrostriction? .............

VI. CONCLUSIONS AND FUTURE PERSPECTIVES . . . . . . . . . . . . . . . .

\section{INTRODUCTION}

Electrostriction is a basic electromechanical phenomenon in all insulators or dielectrics. It describes the electric field/polarization-induced strain $\left(S_{i j}\right)$ that is proportional to the square of electric field $\left(E_{i}\right) /$ polarization $\left(P_{i}\right)$, expressed in the following equations:

$$
\begin{aligned}
S_{i j} & =Q_{i j k l} P_{k} P_{l}, \\
S_{i j} & =M_{i j k l} E_{k} E_{l},
\end{aligned}
$$

where $Q_{i j k l}$ and $M_{i j k l}$ are electrostrictive coefficients. Electrostriction is a four-rank tensor property; thus it can be observed in all crystal symmetries. ${ }^{1}$

The strain induced by the electrostrictive effect is generally small when compared with that induced by piezoelectricity. Consequently, there has been limited attention focusing on the electrostrictive effect. In the 1980s, a systematic study on electrostriction was carried out on relaxor ferroelectrics with perovskite structures, such as $\mathrm{Pb}\left(\mathrm{Mg}_{1 / 3} \mathrm{Nb}_{2 / 3}\right) \mathrm{O}_{3}(\mathrm{PMN})$, $\mathrm{Pb}\left(\mathrm{Zn}_{1 / 3} \mathrm{Nb}_{2 / 3}\right) \mathrm{O}_{3}(\mathrm{PZN})$, and $0.9 \mathrm{~Pb}\left(\mathrm{Mg}_{1 / 3} \mathrm{Nb}_{2 / 3}\right) \mathrm{O}_{3}-0.1 \mathrm{PbTiO}_{3}$ (PMN-0.1PT) single crystals/ceramics, in which a high electrostrictive strain was observed because of the high dielectric response of the relaxors. ${ }^{2-7}$ Relaxors offer several advantages over ferroelectric materials, including low hysteresis in the strain-field response (excellent displacement accuracy), no remnant strain, reduced aging effects, and they do not require poling. ${ }^{3}$ Compared with classical ferroelectric ceramics, these materials are believed to have potential for use in actuator applications such as inchworms, micro-angle adjusting devices, and bistable optical devices, where reproducible and non-hysteretic deformation responses are required. ${ }^{3}$ In addition, in the 1990s, investigations on electrostriction were performed on polymers, ${ }^{8-15}$ including ferroelectric polymers, dielectric elastomers, and polymer composites. Ultra-high electrostrictive strains were observed in these polymeric materials ( $>4 \%$ for polyvinylidene fluoride [PVDF] and $>40 \%$ for silicone), giving them potential for use in actuator applications. ${ }^{15}$

On the other hand, ferroelectrics are the mainstay materials for piezoelectric transducer and actuator applications and have been reported to possess much higher piezoelectric responses when compared with non-ferroelectric materials. ${ }^{16}$ Typical ferroelectric single crystals and ceramics, including barium titanate $\left(\mathrm{BaTiO}_{3}, \mathrm{BT}\right)$ (reported in the 1940s), ${ }^{17}$ lead titanate zirconate $\left(\mathrm{Pb}\left(\mathrm{Zr}_{1-x} \mathrm{Ti}_{x}\right) \mathrm{O}_{3}, \mathrm{PZT}\right)$ polycrystalline ceramics (reported in the 1950s), ${ }^{17}$ and relaxor-PT based single crystals (reported in the 1980 s to 1990 s), ${ }^{18-23}$ exhibited piezoelectric responses that are two to three orders higher than Quartz crystals (first piezoelectric crystal $\sim 2 \mathrm{pC} / \mathrm{N}$ ). Similar to inorganic ferroelectrics, ferroelectric polymers such as PVDF also possess a higher piezoelectric response than their nonferrous counterpart. ${ }^{24,25}$ Thus, it is desirable to understand the origins of high piezoelectric activity in ferroelectrics. For ferroelectrics whose paraelectric phase is centrosymmetric, the piezoelectric coefficient $d_{m i j}$ can be expressed as a derivative of strain to electric field

$$
\begin{aligned}
d_{m i j} & =\frac{\partial S_{i j}}{\partial E_{m}}=\frac{Q_{i j k l} P_{k} \partial P_{l}}{\partial E_{m}}+\frac{Q_{i j k l} P_{l} \partial P_{k}}{\partial E_{m}} \\
& =Q_{i j k l} P_{k} \varepsilon_{l m}+Q_{i j k l} P_{l} \varepsilon_{k m},
\end{aligned}
$$

where $\varepsilon_{i j}$ is the dielectric permittivity, and $i, j, k, l$, and $m=1,2$, or 3. Equation (1) is used as the expression of strain $\left(S_{i j}\right)$. Equation (2) indicates that the piezoelectric coefficients of these ferroelectrics originate from the electrostrictive effect, spontaneous polarization, and the dielectric response. Figure 1 shows that the piezoelectric coefficients can be recognized as the slope of the electrostrictive strain versus the electric field. Thus, we can conclude that the high piezoelectric response in ferroelectrics is established on the electrostrictive effect and is associated with a large "bias polarization" (spontaneous polarization). The electrostrictive effect plays a key role in the electromechanical behavior in ferroelectrics, investigations on which will benefit the exploration of high-performance piezoelectrics. In this paper, the investigations on the electrostrictive effect are surveyed, focusing on ferroelectric-related materials. First, the measurement methods of the electrostrictive effect are given and compared. Second, the origin of electrostriction is discussed for ionic crystals. Then, the relationships between the electrostriction and the crystal structure, dielectric response, elastic property and thermal expansion are surveyed. The electrostrictive properties of the ferroelectric materials are investigated with respect to temperature, composition, ferroelectric phase, and orientation. Finally, the contribution of electrostriction to the piezoelectric activity is discussed in detail for perovskite ferroelectrics, and we outline a possible approach for improving the piezoelectricity through the electrostrictive effect.

\section{THE DETERMINATION OF THE ELECTROSTRICTIVE COEFFICIENTS}

To determine the electrostrictive coefficients, five approaches have been proposed in the literature. These are based on the strain-polarization/electric field curves, dielectric permittivity-stress curves, the piezoelectric effect for the

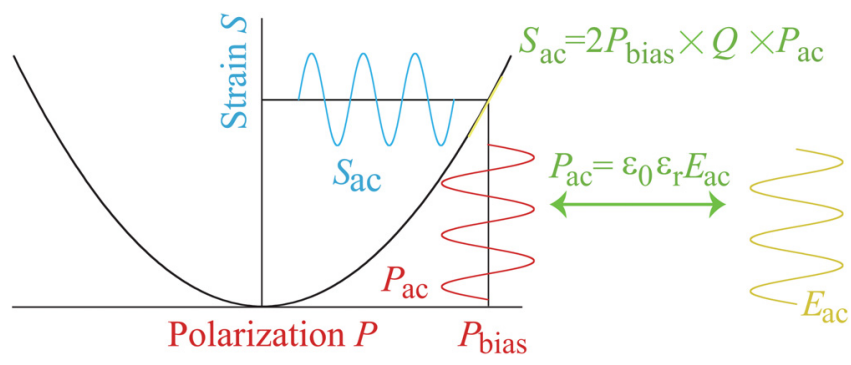

FIG. 1. Schematic plot of the relationship between electrostriction and piezoelectric effects. Due to the high "bias polarization," an $a c$ electric field can induce $a c$ strain with the same frequency of electric field. 

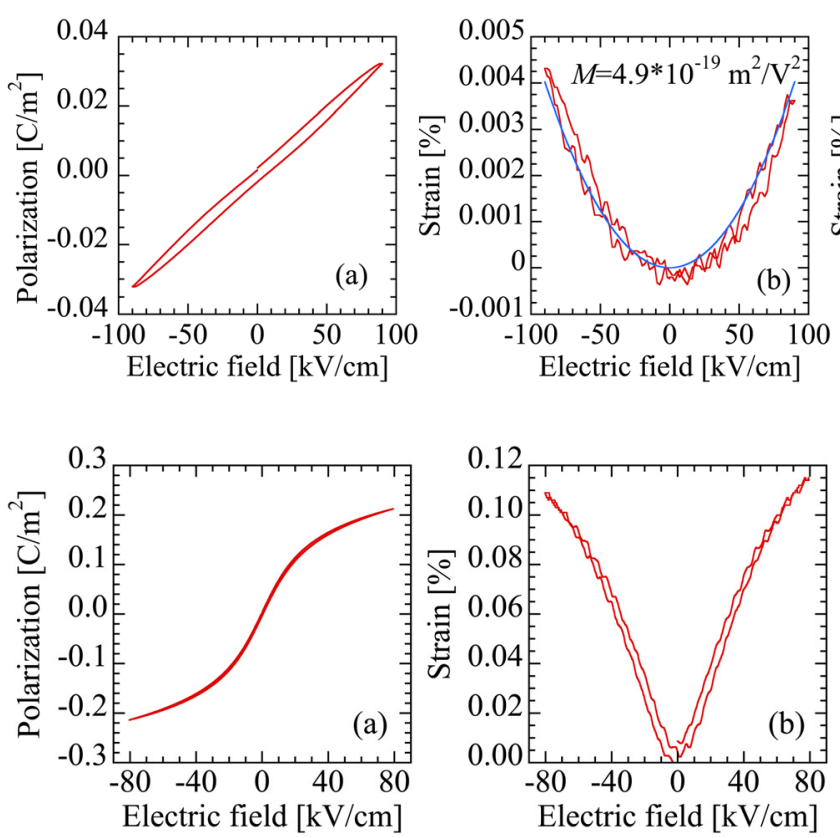

ferroelectrics, the lattice parameters, and the dielectric permittivity under a DC-biased electric field.

\section{A. Electrostrictive coefficients measured by strain versus the polarization/electric field}

According to Eq. (1), the electrostrictive coefficients $Q$ and $M$ can be determined by measuring the strainpolarization (S-P) and strain-electric field (S-E) curves, respectively. The $\mathrm{S}-\mathrm{P}$ and $\mathrm{S}-\mathrm{E}$ curves are accepted as a method to determine the electrostrictive coefficients for materials with a high dielectric response, where the strains can be accurately measured.

Figures 2 and 3 show the measured results for a $\mathrm{BaBi}_{2} \mathrm{Nb}_{2} \mathrm{O}_{9}$ ceramic and an [001] oriented PMN single crystal, respectively. ${ }^{26,27}$ A linear relationship between the polarization and electric field was observed in Fig. 2(a), indicating that the $\mathrm{BaBi}_{2} \mathrm{Nb}_{2} \mathrm{O}_{9}$ ceramic is a linear dielectric material. The strain followed a quadratic function with respect to both the electric-field and polarization, as shown in Figs. 2(b) and 2(c). On the contrary, PMN crystals are nonlinear dielectrics. Figure 3(a) shows the polarization saturation at high fields that deviate from a linear relationship. For nonlinear dielectrics, the electrostrictive strain is not a quadratic function of the electric field but follows a quadratic relation with respect to the polarization (Figs. 3(b) and 3(c)). Thus, the $Q$ coefficient is preferred over the $M$ coefficient, for the investigation of the electrostrictive effect. For this reason, the following discussions on ferroelectrics focus on the $Q$ coefficients.

To determine the $Q$ coefficients in ferroelectrics, domain reorientation (or domain wall motion) and electric fieldinduced phase transitions should be avoided. Figure 4 shows the phenomenon of domain reorientation in tetragonal ferroelectric crystals for the $Q_{33}$ measurements along the [001] direction. For this condition, the contribution of $180^{\circ}$ ferroelectric domain switching contributed to the strain and polarization is $2 d_{33} E$ and $2 P_{s}$, respectively, as shown in Fig. 4(a).

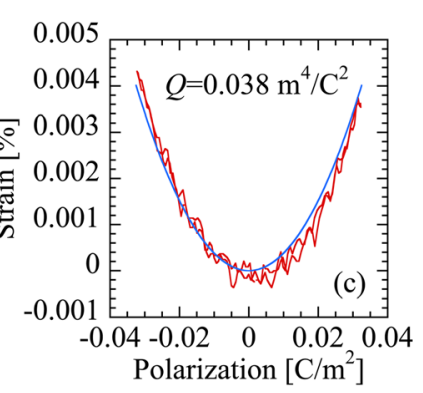

FIG. 2. The polarization-electric field, strain-electric field, and strainpolarization loops for linear dielectrics. Reprinted with permission from G. Viola, T. Saunders, X. Wei, K. B. Chong, H. Luo, M. J. Reece, and H. Yan, J. Adv. Dielectr. 3, 1350007 (2013). Copyright 2013 World Scientific Publishing Company. ${ }^{27}$

FIG. 3. The polarization-electric field, strain-electric field, and strainpolarization loops for nonlinear dielectrics (taking [001] oriented PMN crystal as example).
Generally, $2 d_{33} E$ was much smaller than $4 P_{S}^{2} Q_{33}$; thus the presence of the $180^{\circ}$ ferroelectric domain switching mitigates the measured $Q$ value. On the contrary, the $90^{\circ}$ ferroelastic domain switching made the measured $Q_{33}$ larger than the real value of the lattice deformation. This was caused by polarization variations along the perpendicular direction, which was not considered in the measurement. Overall, domain switching impacted the electrostrictive coefficient measurements. Ferroelectric phase transitions also affected the measured results, because the direction of the spontaneous polarization varied during the ferroelectric phase transitions. To eliminate the impact of the domain wall motion
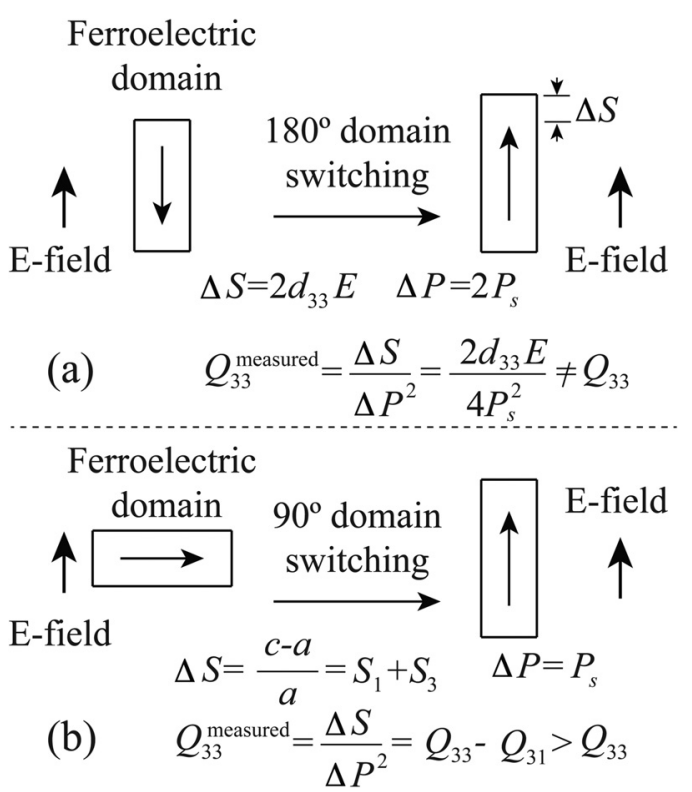

FIG. 4. Schematic figure of domain reorientation effect on determination of longitudinal electrostrictive coefficient $Q_{33}$, where $90^{\circ}$ and $180^{\circ}$ domain reorientations are considered. In figure (a), the $d_{33}, E$, and $P_{\mathrm{S}}$ are piezoelectric coefficient, coercive field, and spontaneous polarization, respectively. The $S_{3}$ and $S_{1}$ are the spontaneous strain along $c$ and $a$ crystallographic axes, respectively. 


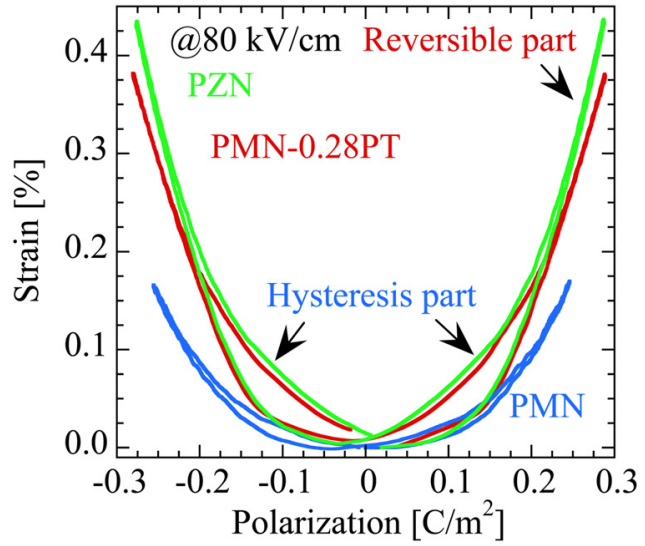

FIG. 5. The strain-polarization loops for [001] oriented PMN, PMN-0.28PT, and PZN crystals, being measured at 25,150 , and $150^{\circ} \mathrm{C}$, respectively. Reprinted with permission from Appl. Phys. Lett. 102, 152910 (2013). Copyright 2013 AIP Publishing LLC. ${ }^{26}$

and phase transition, the S-P curves should be measured at high temperatures (higher than the Curie temperature, $T_{C}$ ) and at high electric fields, because no ferroelectric domains exist in the paraelectric phase above the $T_{C}$, meanwhile, the domains could be clamped by high electric fields, increasing the difficulty in the domain switching.

Figure 5 shows the electrostriction results for [001] oriented PMN, PMN-0.28PT, and PZN crystals at temperatures above the temperature of maximum permittivity $\left(T_{m}\right){ }^{26}$ The S-P curves exhibited hysteretic behavior, which can be separated into hysteretic and reversible regions. The hysteretic region in the S-P curves can be attributed to either the microdomain reorientation in relaxor materials (micro-domains exist at temperatures above $T_{m}$ ) or the electric-field-induced micro-macro domain transition. ${ }^{28,29}$ It should be noted here that the hysteretic region was also related to the measurement condition. For example, PMN-0.1PT thin film does not show obvious hysteretic S-P behavior at the temperatures above $T_{m} \cdot{ }^{30}$ The difference between the results of Fig. 5 and Ref. 30 can be attributed to the substrate clamping effect of thin film and different measurement conditions (frequency and amplitude of electric field). The strain and polarization induced by the intrinsic lattice contribution at a high electric field accounts for the reversible S-P curve. By fitting the experimental data using $S_{33}=Q_{33} P_{3}^{2}$, the electrostrictive coefficient $Q_{33}$ can be determined with respect to the amplitude of the electric field, as given in Fig. 6. In the electric field range of $10-80 \mathrm{kV} / \mathrm{cm}$, the measured $Q_{33}$ values for [001] oriented PMN-0.28PT, PZN, and PMN crystals increased with the electric field increasing, being in the ranges of $0.037-0.053 \mathrm{~m}^{4} / \mathrm{C}^{2}, 0.043-0.060 \mathrm{~m}^{4} / \mathrm{C}^{2}$, and $0.018-0.027$ $\mathrm{m}^{4} / \mathrm{C}^{2}$, respectively. This was because the micro-domain reorientation $\left(180^{\circ}\right)$ reduced the measured $Q$ value at low electric fields, while the measured $Q$ approached the intrinsic value (lattice) at high electric fields.

\section{B. Electrostrictive coefficients measured using the dielectric permittivity versus the applied stress}

Determination of electrostrictive coefficients from the relationship between dielectric permittivity and applied stress was based on the converse effect of electrostriction. The direct effect of electrostriction was given in Eq. (1) from which the electrostrictive coefficient $Q_{i j k l}$ can be expressed as follows:

$$
Q_{i j k l}=\frac{1}{2} \frac{\partial^{2} S_{i j}}{\partial P_{k} \partial P_{l}} .
$$

Based on the elastic Gibbs free energy [Eq. (4)] and changing the order of differentiation in the free energy equation, Eq. (3) was transformed to Eq. (5) (Refs. 31 and 32)

$$
\begin{gathered}
d G_{1}=-S_{i j} d X_{i j}+E_{i} d P_{i}-S d T \\
Q_{i j k l}=-\frac{1}{2} \frac{\partial^{3} G_{1}}{\partial X_{i j} \partial P_{k} \partial P_{l}}=-\frac{1}{2} \frac{\partial^{2} E_{k}}{\partial X_{i j} \partial P_{l}}=-\frac{1}{2} \frac{\partial \chi_{k l}}{\partial X_{i j}},
\end{gathered}
$$

where $G_{1}$ is the elastic Gibbs free energy, $X_{i j}$ is the stress, $S$ is the entropy, $T$ is the temperature, and $\chi_{k l}$ is the inverse dielectric permittivity. Using the same method, the coefficient $M_{i j k l}$ can be expressed as

$$
\begin{gathered}
d G=-S_{i j} d X_{i j}-P_{i} d E_{i}-S d T \\
M_{i j k l}=-\frac{1}{2} \frac{\partial^{3} G}{\partial X_{i j} \partial E_{k} \partial E_{l}}=\frac{1}{2} \frac{\partial^{2} P_{k}}{\partial X_{i j} \partial E_{l}}=-\frac{1}{2} \frac{\partial \varepsilon_{k l}}{\partial X_{i j}}
\end{gathered}
$$

where $G$ is the Gibbs free energy and $\varepsilon_{k l}$ is the dielectric permittivity. Equations (5) and (7) describe the converse effect of
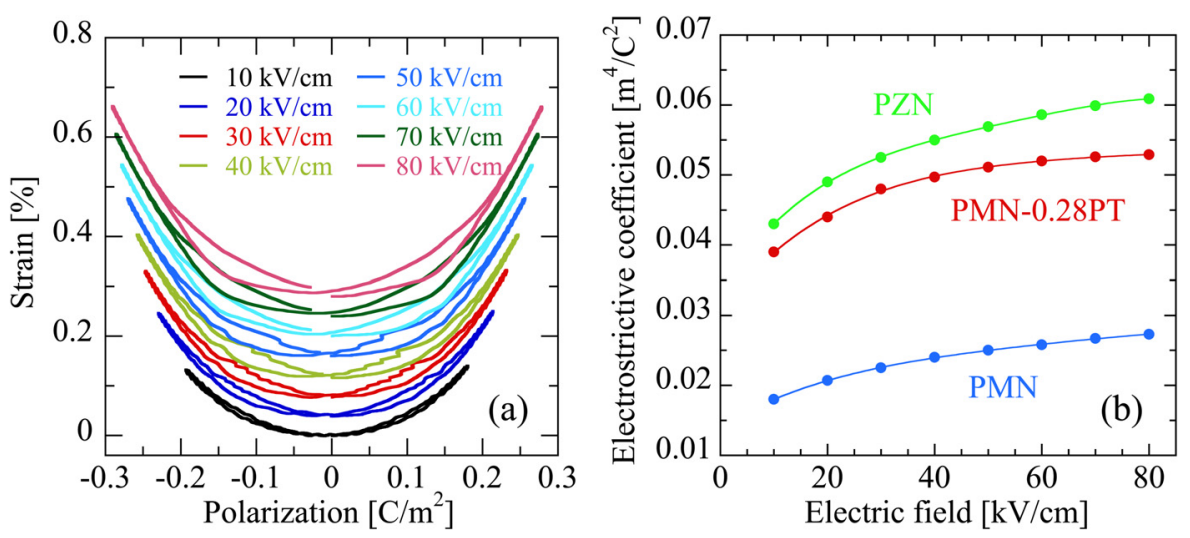

FIG. 6. (a) Strain-polarization loops for [001] oriented PMN-0.28PT measured at various amplitudes of electric field. (b) The electrostrictive coefficient $Q_{33}$ with respect to the amplitude of electric field for [001] oriented PMN, PMN-0.28PT, and PZN crystals. Reprinted with permission from Appl. Phys. Lett. 102, 152910 (2013). Copyright 2013 AIP Publishing LLC. ${ }^{26}$ 


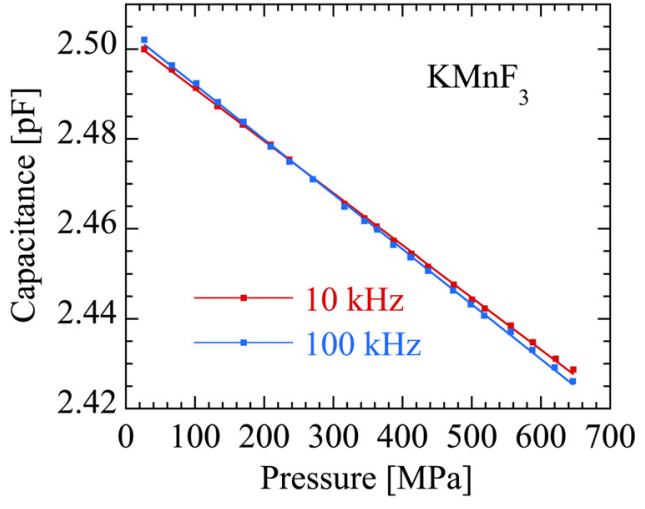

FIG. 7. Determination of $Q_{h}$ for $\mathrm{KMnO}_{3}$ via converse electrostrictive effect. Reprinted with permission from K. Rittenmyer, A. S. Bhalla, and L. E. Cross, Ferroelectrics Lett. Sec. 9, 161 (1989). Copyright 1989 Taylor \& Francis. ${ }^{33}$

electrostriction. According to Eq. (5), the $Q$ coefficients can be written as follows:

$$
\begin{aligned}
Q_{33} & =-\frac{1}{2} \frac{\partial \chi_{33}}{\partial X_{33}}, \\
Q_{31} & =-\frac{1}{2} \frac{\partial \chi_{11}}{\partial X_{33}} .
\end{aligned}
$$

In addition, for crystals with cubic symmetry, the hydrostatic electrostrictive coefficient $Q_{h}$ can be written as ${ }^{33}$

$$
Q_{h}=-\frac{1}{2} \frac{\partial \chi_{11}}{\partial p}
$$

where $p$ is the applied hydrostatic pressure. Equations (8)-(10) were successfully employed to determine the $Q$ coefficients for materials with low electrostrictive strains. In these materials, it was difficult to accurately measure the S-P curves. Based on this approach, the $Q_{h}$ coefficient for $\mathrm{KMnO}_{3}$ was determined, as shown in Fig. 7. ${ }^{11,33}$

It is important to note that Eqs. (4)-(10) are only applicable for linear systems. In ferroelectric materials, the inverse dielectric permittivity and stress may not follow a linear relationship because of nonlinear phenomena such as the depolarization, domain wall motion, and phase transitions. ${ }^{32}$ Thus, the effects of the nonlinear phenomena should be minimized under the applied stress/hydrostatic pressure to accurately measure the $Q$ coefficients in ferroelectrics. Figure 8 presents the stressdependent dielectric properties for PMN- $x$ PT crystals. ${ }^{34,35}$ In single domain crystals, the stress perpendicular to the poling direction was found to stabilize the polarization state; at this time, the depolarization or domain wall motion was difficult to be induced. For this condition, the measured $Q_{33}$ coefficients were almost the same values as those measured from the S-P curves, as shown in Figs. 8(a) and 8(b). On the contrary, strong nonlinear phenomena existed when the compressive stress was along the poling direction, where a drastic increase in $\chi_{33}$ was caused by depolarization of the crystal, as shown in Fig. 8(c). For the case of hydrostatic pressure, $\chi_{k l}$ and the pressure followed a linear relationship at low pressures, and the obtained $Q_{h}$ was close to that derived from the piezoelectricity of the crystal. At high level of hydrostatic pressure, however, ferroelectric phase transition may be induced, leading to nonlinear characteristics; the measured $Q_{h}$ deviates from the real value; therefore, great attention needs to be paid to this method for determination of electrostrictive coefficients of ferroelectrics.

\section{Electrostrictive coefficients determined using the piezoelectric coefficients}

For ferroelectric single crystals, the electrostrictive coefficients can be calculated from the piezoelectric coefficients, dielectric permittivities, and spontaneous polarization, based on Eq. (2). For perovskite crystals with $3 m, m m 2$, and $4 m m$
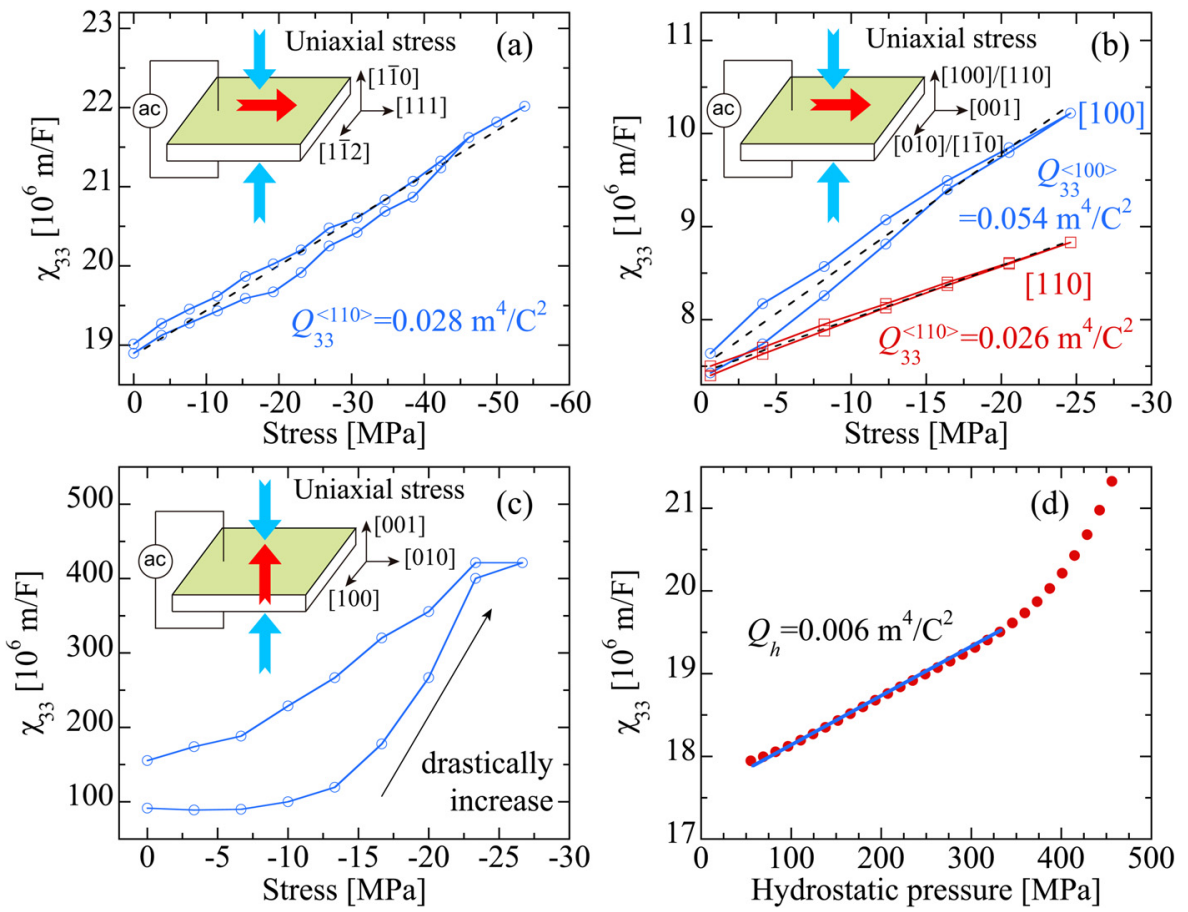

FIG. 8. Stress dependence of inverse dielectric permittivity for PMN-xPT crystals. (a) The stress along [110] direction was applied to [111] poled PMN-0.28PT rhombohedral crystals. The coefficient $Q_{33}$ along $\langle 110\rangle$ direction can be determined. (b) The stresses along [100] and [110] directions were applied to [001] poled PMN-0.37PT tetragonal crystals. The coefficient $Q_{33}$ along $\langle 100\rangle$ and $\langle 110\rangle$ directions can be obtained. (c) The stress along [001] direction was applied to [001] poled PMN-0.37PT tetragonal crystals. (d) The hydrostatic pressure dependence of inverse dielectric permittivity for [001] poled PMN-0.28PT. The red arrows represent the poling/single domain directions of the crystals. The blue arrows represent the direction of the applied stress. The dash lines are the fitting slope of reciprocal susceptibility $\chi_{33}$ vs. stress. 
symmetries, detailed information on their calculations is given in the Appendix.

With this method, high temperature and high field (electric field or stress) measurements were not required. The full set of electrostrictive coefficients can be easily determined because the full set of piezoelectric coefficients can be derived using conventional approaches, such as resonance, ultrasonic, and the Berlincourt methods. ${ }^{36}$ For ferroelectric ceramics, reliable information could not be derived using this method because the extrinsic contributions of the piezoelectric and dielectric properties from the domain wall motion (which is a nonlinear phenomenon) are quite high, being $10 \%-40 \%$. Of particular importance is that the domain walls can be eliminated by poling along the direction of the spontaneous polarization in ferroelectric crystals. Thus, this method was preferred for ferroelectric crystals rather than ceramics.

\section{Electrostrictive coefficients determined from the lattice parameters}

The spontaneous strain in ferroelectrics can be inferred from the lattice parameters. The $Q$ coefficients can be obtained as the ratio between the spontaneous strain and the square of the spontaneous polarization. Berlincourt and Jaffe provided reliable $Q$ coefficients for BT crystal with this method $^{37}$

$$
\begin{aligned}
& Q_{33}=S_{3} / P_{3}^{2}=0.0075 / 0.26^{2}=0.111 \mathrm{~m}^{4} / \mathrm{C}^{2}, \\
& Q_{31}=S_{1} / P_{3}^{2}=0.0030 / 0.26^{2}=0.044 \mathrm{~m}^{4} / \mathrm{C}^{2},
\end{aligned}
$$

where $Q_{33}$ and $Q_{31}$ are the electrostrictive coefficients for the BT crystals (in the standard coordinate system with $4 \mathrm{~mm}$ symmetry), $S_{3}$ and $S_{1}$ are spontaneous strains, and $P_{3}$ is the spontaneous polarization.

Based on this notation, the $Q_{i j k l}$ coefficients for PZT and doped PMN single crystals were determined, where the lattice parameters were measured by powder diffraction (either X-ray or neutron diffraction). ${ }^{38-41}$ The nonlinear phenomena were neglected in this approach as no electric field or stress was applied for this method.

\section{E. Electrostrictive coefficients determined from the dielectric permittivity under a DC-biased electric field}

Based on the theoretical Debye model, Pattnaik and Toulouse developed an expression for the dielectric permittivity involving the electrostrictive coefficient $M$ with a cubic symmetry, as given in Eqs. (13) and (14) (Ref. 42)

$$
\begin{gathered}
\varepsilon^{\prime}(\omega)=\frac{\varepsilon_{s}+\varepsilon_{\infty} \omega^{2} \tau^{2}}{\omega^{2} \tau^{2}+1}-\frac{1}{s_{11}^{E}} \frac{4 E_{0}^{2} M_{13}^{2}}{\omega^{2} \tau^{2}+1}\left(1-Y_{r}-\omega \tau Y_{i}\right), \\
\varepsilon^{\prime \prime}(\omega)=\frac{\left(\varepsilon_{s}-\varepsilon_{\infty}\right) \omega \tau}{\omega^{2} \tau^{2}+1}-\frac{1}{s_{11}^{E}} \frac{4 E_{0}^{2} M_{13}^{2}}{\omega^{2} \tau^{2}+1}\left(\omega \tau-\omega \tau Y_{r}+Y_{i}\right),
\end{gathered}
$$

where $\varepsilon^{\prime}(\omega)$ and $\varepsilon^{\prime \prime}(\omega)$ are the real and imaginary dielectric permittivities, respectively, $\varepsilon_{s}$ and $\varepsilon_{\infty}$ are the static and infinite frequency limits of the permittivities, respectively, $\omega$ is the angular frequency, $\tau$ is the relaxation time, $s_{11}^{E}$ is the elastic constant, $E_{0}$ is the DC bias field, $Y_{r}$ and $Y_{i}$ are proportional to the mechanical strain. Detailed information on the coefficients $Y_{r}$ and $Y_{i}$ are given in Ref. 42. The electrostrictive coefficient $M_{13}$ can be extracted using Eqs. (13) and (14). ${ }^{42,43}$ The issue with this method was whether or not a simple Debye model is available for the studied dielectrics.

In this section, the experimental methods for determining the electrostrictive coefficients were surveyed. The methods used to determine the electrostrictive coefficients are summarized in Table I, where the characteristics and issues with each method are presented. It should be noted that the electrostrictive coefficients for ferroelectrics can also be determined using a first-principle approach, where the calculated electrostrictive coefficients for $\mathrm{LiNbO}_{3}$ and $\mathrm{BaTiO}_{3}$ crystals were reported to be in good agreement with the measured results. ${ }^{44,45}$

\section{THE ORIGIN OF ELECTROSTRICTION}

Electrostriction is present in all crystal symmetries because it is a fourth rank polar tensor. Electrostriction is a measure of the polarization induced by ions shifting away from their natural equilibrium positions, giving rise to variations in the lattice parameters (strain). In centrosymmetric crystals, the induced shifts of equivalent ions almost cancel each other out, while the difference in the shifts because of potential anharmonicity generates strain. ${ }^{3}$ The typical potential of an ion-pair is shown in Fig. 9, where the energy required to compress the ion-pair $\left(U_{C}\right)$ is higher than the energy required to extend the ion pair $\left(U_{E}\right){ }^{46,47}$ Based on the potential characteristics, the relative displacement between positive and negative ions (polarization) is prone to occur via extension of the lattice instead of compression, as shown in Fig. 10. As a consequence, the longitudinal electrostrictive coefficient $Q_{33}$ is generally positive for ionic crystals. ${ }^{48}$

A quantitative analysis on the above phenomenon, proposed by Uchino et al., ${ }^{49}$ is presented in this section. The potential energy of the ion pair is the simplest rigid ion model with $+\mathrm{q}$ and $-\mathrm{q}$ ions and can be written as

$$
\Delta U=U(r)-U\left(r_{0}\right)=f\left(r-r_{0}\right)^{2}-g\left(r-r_{0}\right)^{3},
$$

where $r$ is the distance between a cation and its nearestneighbor anion and $r_{0}$ is the equilibrium position. $U(r)$ and $U\left(r_{0}\right)$ are the potentials for the nearest neighbors separated by distances of $r$ and $r_{0}$, respectively. The first and second terms in the energy potential are the harmonic and anharmonic components, respectively, where the high order terms were ignored. The coefficients $f$ and $g$ are associated with the crystal structure and the characteristics of the ions (charge and radius). Under an electric field, the average induced strain $\left\langle x_{1}\right\rangle$ and polarization $P_{1}$ can be calculated using Boltzmann distribution

$$
\begin{gathered}
\left\langle x_{1}\right\rangle=\left(3 g q^{2} / 4 f^{3} r_{0}\right) E_{1}^{2}, \\
P_{1}=\left(q^{2} / 4 f r_{0}^{3}\right) E_{1},
\end{gathered}
$$




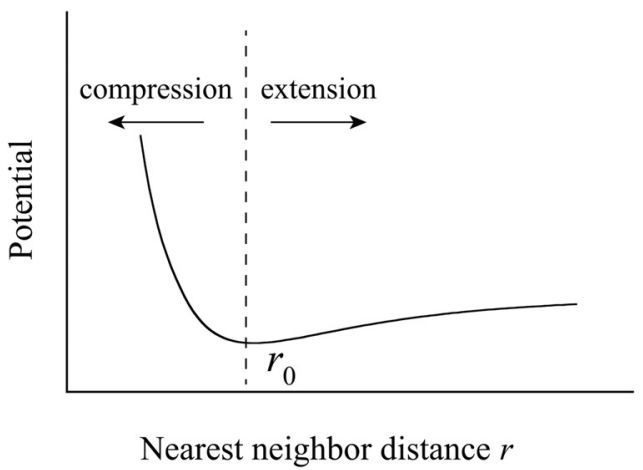

FIG. 9. Schematic figure of the pair potential in ionic crystal, where $r_{0}$ represents the equilibrium distance between positive and negative ions.

where $q$ is the charge of the ions. Consequently, the electrostrictive coefficient $Q_{11}$ is given by

$$
Q_{11}=12 g r_{0}^{5} / f q^{2} .
$$

It can be seen from Eq. (18) that a high $g / f$ corresponds to a high $Q_{11}$, where the $g / f$ ratio represents the comparison between the anharmonic and harmonic energy components. The coefficients $f$ and $g$ are both positive; thus, the longitudinal coefficient $Q_{11}$ is also a positive coefficient.

Similar to the electrostrictive effect, Maxwell stress contributes to the strain, which is also proportional to the square of the electric field/polarization. As an electric field is applied to the electrodes on the dielectrics, the electrostatic force resulting from the free charges squeezes or stretches the materials. This electrostatic force is called the Maxwell stress and occurs in all insulators with an electric field applied to the deposited electrodes. ${ }^{50}$ The longitudinal strain for dielectrics induced by a Maxwell stress is given by

$$
S_{M}=-\frac{1}{2} s \varepsilon E^{2},
$$

where $S_{M}$ is the longitudinal strain induced by the Maxwell stress, $s$ is the elastic compliance of the material, $\varepsilon$ is the dielectric permittivity. The difference between the strains induced by electrostriction and the Maxwell stress is that the former is caused by the potential anharmonicity of the ionic crystal, while the latter originates from free charges on the electrodes. However, in S-E loop measurements, these two

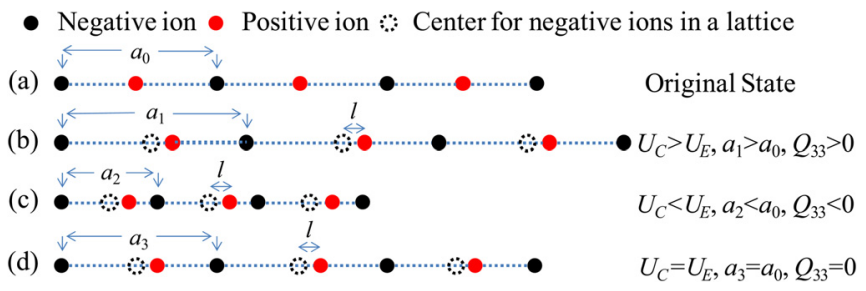

FIG. 10. Rigid model of ionic crystal to illustrate the electrostrictive effect. The lattice parameter variations are given under three potential energy conditions, for the same relative displacement between positive and negative ions (noted as $l$ ). (a) original state for crystal with zero polarization; (b) in the case of $U_{C}>U_{E}, l$ is induced by the extension of lattice; (c) in the case of $U_{C}<U_{E}, l$ is induced by the compression of lattice; (d) in the case of $U_{C}=U_{E}, l$ exists without varying the lattice parameter. 
contributions are generally mixed with each other. When determining the electrostrictive coefficient $Q$, if the strain induced by Maxwell stress is not separated from the total strain, the contribution of the Maxwell stress to the apparent electrostrictive coefficients $\left(M_{M}\right.$ or $\left.Q_{M}\right)$ can be written as follows:

$$
\begin{aligned}
& M_{M}=\frac{S_{M}}{E^{2}}=-\frac{1}{2} s \varepsilon, \\
& Q_{M}=\frac{M_{M}}{\varepsilon^{2}}=-\frac{1}{2} \frac{s}{\varepsilon} .
\end{aligned}
$$

It can be seen from Eqs. (20) and (21) that the longitudinal electrostrictive coefficient induced by the Maxwell stress is negative. The contribution of the Maxwell stress is significant in materials with either a large elastic compliance (such as soft polymers) or a low dielectric constant (such as $\mathrm{CaF}_{2}$ ) [as expressed in Eq. (21)], ${ }^{11}$ while it can be ignored in ferroelectric crystals and ceramics.

\section{ELECTROSTRICTION WITH RESPECT TO THE MICROSCOPIC AND MACROSCOPIC CHARACTERISTICS}

The values of the electrostrictive coefficients for various dielectrics span many orders of magnitude. The longitudinal coefficients $Q_{33}$ and $Q_{11}$ for soft elastomers are on the order of $10^{4} \mathrm{~m}^{4} / \mathrm{C}^{2}$, while those for perovskite ferroelectrics are about $10^{-1}$ to $10^{-2} \mathrm{~m}^{4} / \mathrm{C}^{2}$, as listed in Table II. ${ }^{11,37,51-56}$ The electrostrictive $Q$ coefficients have been analyzed with respect to the microstructures and macroscopic properties of the materials.

In this section, we focus on the hydrostatic electrostrictive coefficients $Q_{h}$, because the available $Q_{h}$ data for various dielectrics were more comprehensive in comparison with other coefficients (such as $Q_{11}, Q_{12}$ ).

\section{A. Microscopic characteristics versus the electrostrictive effect}

The microscopic characteristics, including the ionic radius, ionic charge, and crystal structure, are believed to be the dominant factors for electrostriction. As listed in Table II, the crystals with similar microscopic characteristics exhibited similar $Q$ values. In the simplest rigid model, the effect of the microscopic characteristics can be symbolized by the coefficients $r_{0}, f, g$, and $q$ in Eq. (18). For crystals with the formulas $\mathrm{ABO}_{3}$ and $\mathrm{ABF}_{3}$, the coefficients $r_{0}, f$, and $g$ were assumed to be of the same order because they have the same perovskite structure. Thus, the difference in the $Q$ values between the $\mathrm{ABO}_{3}$ and $\mathrm{ABF}_{3}$ crystals was dominated by the difference in the valences $q$ of the ions. The valence

TABLE II. The electrostrictive coefficients for various types of dielectrics. (The electrostrictive coefficients of crystals are measured in their respective stand-

\begin{tabular}{|c|c|c|c|c|c|}
\hline Oxygen perovskite & & $Q_{11}$ & $Q_{12}$ & $Q_{44}$ & References \\
\hline \multirow{2}{*}{$\begin{array}{l}\text { Measured in the coordinate system, } \\
\text { whose } X, Y \text {, and } Z \text { axes are along [100], } \\
{[010] \text {, and [001] directions, respectively }}\end{array}$} & $\mathrm{BaTiO}_{3}$ & 0.11 & -0.045 & 0.059 & 37 and 45 \\
\hline & $\mathrm{PbTiO}_{3}$ & 0.09 & -0.03 & 0.034 & 51 \\
\hline \multicolumn{2}{|l|}{ Ilmenite } & $Q_{33}$ & $Q_{31}$ & $Q_{44}$ & 52 and 53 \\
\hline \multirow{2}{*}{$\begin{array}{l}\text { Measured in the coordinate system, } \\
\text { whose } X, Y \text {, and } Z \text { axes are along }[1 \overline{1} 0] \text {, } \\
{[11 \overline{2}] \text {, and }[111] \text { directions, respectively }}\end{array}$} & $\mathrm{LiNbO}_{3}$ & 0.016 & -0.003 & 0.065 & 52 and 53 \\
\hline & $\mathrm{LiTaO}_{3}$ & 0.021 & -0.005 & 0.059 & 52 and 53 \\
\hline \multirow{4}{*}{$\begin{array}{l}\text { Fluorite structure } \\
\text { Measured in the coordinate system, } \\
\text { whose } \mathrm{X}, \mathrm{Y} \text {, and } \mathrm{Z} \text { axes are along [100], } \\
\text { [010], and [001] directions, respectively }\end{array}$} & & $Q_{11}$ & $Q_{12}$ & $Q_{44}$ & 11 and 54 \\
\hline & $\mathrm{CaF}_{2}$ & -0.48 & -0.48 & 1.99 & 11 and 54 \\
\hline & $\mathrm{BaF}_{2}$ & -0.33 & -0.29 & 1.46 & 11 and 54 \\
\hline & $\mathrm{SrF}_{2}$ & -0.33 & -0.39 & 1.90 & 11 and 54 \\
\hline \multicolumn{2}{|l|}{ Fluoride perovskites } & $Q_{11}$ & $Q_{h}$ & & 55 \\
\hline \multirow{3}{*}{$\begin{array}{l}\text { Measured in the coordinate system, } \\
\text { whose } X, Y \text {, and } Z \text { axes are along [100], } \\
{[010] \text {, and [001] directions, respectively }}\end{array}$} & $\mathrm{KMnF}_{3}$ & 0.51 & 0.22 & & 55 \\
\hline & $\mathrm{KMgF}_{3}$ & $\cdots$ & 0.24 & & 55 \\
\hline & $\mathrm{KZnF}_{3}$ & $\ldots$ & 0.28 & & 55 \\
\hline \multirow[t]{4}{*}{ Sodium chloride structure } & & $Q_{11}$ & $Q_{h}$ & & 49 and 56 \\
\hline & $\mathrm{LiF}$ & 0.57 & 0.32 & & 49 and 56 \\
\hline & $\mathrm{NaF}$ & $\ldots$ & 0.46 & & 49 and 56 \\
\hline & $\mathrm{NaCl}$ & $\ldots$ & 1.44 & & 49 and 56 \\
\hline \multirow[t]{3}{*}{$P V D F$} & & $Q_{11}$ & $Q_{h}$ & & \\
\hline & PVDF & $-12^{\mathrm{a}}$ & -2.4 & & 11 \\
\hline & $\mathrm{P}(\mathrm{VDF}-\mathrm{TrFE})$ & $-13^{\mathrm{a}}$ & -2.5 & & 8 \\
\hline \multirow[t]{3}{*}{ Elastomers } & & $Q_{11}$ & $Q_{h}$ & & \\
\hline & Polyurethane & $-1100^{\mathrm{a}}$ & -850 & & 14 \\
\hline & fluoroelastomer & $-6200^{\mathrm{a}}$ & $\ldots$ & & 14 \\
\hline
\end{tabular}
ard coordinate systems.) Unit of $Q$ is $\mathrm{m}^{4} / \mathrm{C}^{2}$.

${ }^{\text {a The }} Q$ data for polymers are calculated by equation $Q_{11}=S / P^{2}$, which is the apparent electrostrictive coefficient including the contribution of Maxwell stress. $^{14}$ 
TABLE III. The hydrostatic electrostrictive coefficients and Curie constant for perovskite materials. Unit of $Q_{h}$ is $\mathrm{m}^{4} / \mathrm{C}^{2}$, unit of $C$ is $10^{5} \mathrm{~K}$, and unit of $Q_{h} C$ is $10^{3} \mathrm{~m}^{4} \mathrm{~K} / \mathrm{C}^{2}$.

\begin{tabular}{|c|c|c|c|c|c|c|}
\hline Polar type & Order type & Materials & $Q_{h}$ & $C$ & $Q_{h} C$ & References \\
\hline \multirow[t]{4}{*}{ Ferroelectric } & Order & $\mathrm{BaTiO}_{3}$ & 0.02 & 1.5 & 3.0 & 37 \\
\hline & & $\mathrm{PbTiO}_{3}$ & 0.022 & 1.7 & 3.7 & 51 \\
\hline & & $\mathrm{SrTiO}_{3}$ & 0.047 & 0.77 & 3.6 & 62 \\
\hline & & $\mathrm{KTaO}_{3}$ & 0.052 & 0.5 & 2.6 & 63 \\
\hline \multirow[t]{3}{*}{ Relaxor } & Partially order & $\mathrm{Pb}\left(\mathrm{Sc}_{1 / 2} \mathrm{Ta}_{1 / 2}\right) \mathrm{O}$ & 0.0083 & 3.5 & 2.9 & 64 \\
\hline & Disorder & $\mathrm{PMN}$ & 0.006 & 4.7 & 2.8 & 2 \\
\hline & & PZN & 0.0066 & 4.7 & 3.1 & 65 \\
\hline \multirow[t]{2}{*}{ Antiferro } & Order & $\mathrm{PbZrO}_{3}$ & 0.02 & 1.6 & 3.2 & 66 \\
\hline & & $\mathrm{Pb}\left(\mathrm{Mg}_{1 / 2} \mathrm{~W}_{1 / 2}\right) \mathrm{O}_{3}$ & 0.062 & 0.42 & 2.6 & 67 \\
\hline \multirow[t]{4}{*}{ Nonpolar } & Order & $\mathrm{BaZrO}_{3}$ & 0.023 & & & 7 \\
\hline & Disorder & $\left(\mathrm{K}_{3 / 4} \mathrm{Bi}_{1 / 4}\right)\left(\mathrm{Zn}_{1 / 6} \mathrm{Nb}_{5 / 6}\right) \mathrm{O}_{3}$ & 0.0055 & & & 7 \\
\hline & & $0.856 \mathrm{SrTiO}_{3}-0.144 \mathrm{Bi}_{2 / 3} \mathrm{TiO}_{3}$ & 0.013 & & & 7 \\
\hline & & $\mathrm{K}_{0.5} \mathrm{Na}_{0.5} \mathrm{NbO}_{3}-\mathrm{SrTiO}_{3}$ & $0.004-0.007$ & & & 79 \\
\hline
\end{tabular}

ratio between $\mathrm{F}^{-}$and $\mathrm{O}^{2-}$ was 1:2. Thus, the electrostrictive coefficient for $\mathrm{ABF}_{3}$ was expected to be about four times larger than that for $\mathrm{ABO}_{3}$, which is in good agreement with the measured results listed in Table II. Sundar and Newnham presented detailed expressions for the coefficients $f$ and $g$ according to the Born-Mayer potential with which the electrostrictive coefficient $Q$ was accurately calculated for a $\mathrm{LiF}$ crystal giving the value of $0.53 \mathrm{~m}^{4} / \mathrm{C}^{2}$, which is consistent with the experimental result. ${ }^{57}$

The effects of the microscopic characteristics on the electrostrictive coefficient $Q$ were further demonstrated with the degree order of the B-site cations in perovskite materials. Table III shows that the magnitude of the electrostrictive coefficient strongly depends on the level of orderdisorder in the B-site cation arrangement. It was concluded that the electrostrictive coefficient $Q$ increased with the order level of the B-site cations, from disordered to partially ordered, and then to ordered structures. A schematic crystallographic model, proposed by Uchino, can be used to explain this phenomenon (Fig. 11). ${ }^{3}$ In this model, a large "rattling" space was expected for the smaller B-site cations (B2) in the disordered structures, because the larger B-site cations (B1) prop open the lattice framework. Meanwhile, less "rattling" space was expected in the ordered arrangement where the neighboring oxygen atoms were densely packed around the smaller B ions (B2). When an electric field was applied to the disordered perovskites, the B2 cations with a large rattling space could easily shift without distorting the octahedron framework. Therefore, a larger polarization and lower strains were expected for unit magnitude of the electric field, resulting in smaller electrostrictive coefficients and larger dielectric permittivities. On the contrary, in the ordered perovskites with a very small rattling space, the B2 ions could not move without distorting the octahedron, leading to a smaller dielectric permittivity and larger electrostrictive coefficients.

To obtain accurate electrostrictive coefficients from the microscopic characteristics, more complicated models have been proposed. ${ }^{58-62}$ Though microscopic characteristics are the dominant factor for the $Q$ values, the relationship between the electrostriction and macroscopic properties, such as the dielectric properties, elastic properties, and thermal expansion, were also established for evaluation of the electrostriction.

\section{B. Macroscopic characteristics versus the electrostrictive effect}

\section{Dielectric and elastic responses versus the electrostrictive effect}

Figure 12 shows $\log \left(Q_{h}\right)$ as a function of $\log (s / \varepsilon)$ for various materials, including ceramics and polymers, where a linear relationship was observed, demonstrating that the electrostrictive coefficient $Q_{h}$ was proportional to $s / \varepsilon$. Using a simple atomistic model, Newnham et al. ${ }^{1,11}$ proposed a relationship between dielectric/elastic and the electrostrictive

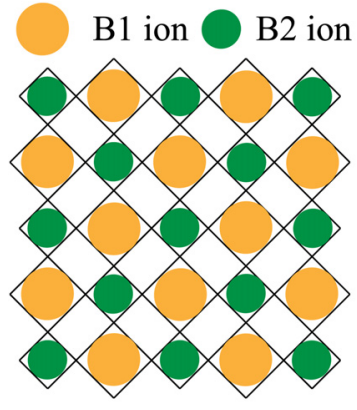

(a)

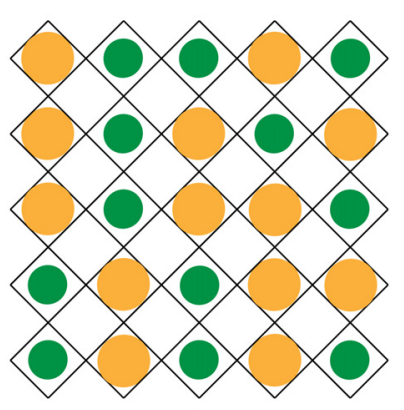

(b)

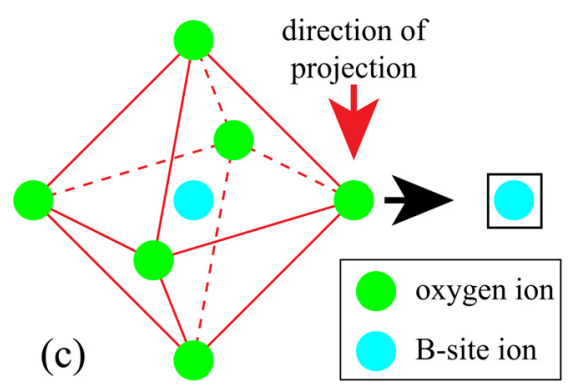

FIG. 11. A tentative crystallographic model, showing order and disorder structures in perovskite crystals. (a) The condition of B-site cations ordered; (b) the condition of B-site cations disordered; (c) the projection of oxygenoctahedron, where the oxygen ions are not plotted in the figure. Reprinted with permission from K. Uchino, S. Nomura, L. E. Cross, R. E. Newnham, and S. J. Jang, J. Mater. Sci. 16, 569 (1981). Copyright 1981 Springer. $^{3}$ 


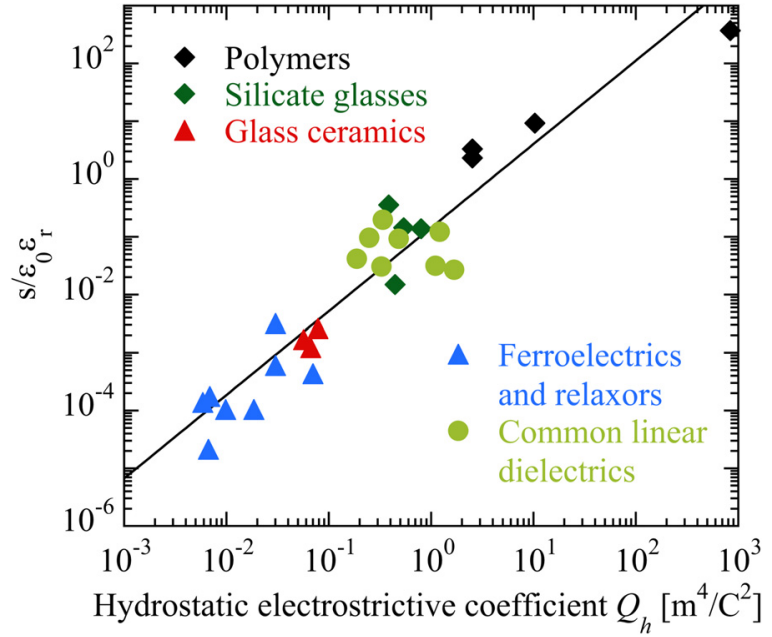

FIG. 12. Hydrostatic electrostrictive coefficient $Q_{h}$ as a function of the ratio of elastic compliance to dielectric permittivity for dielectrics with large elastic compliance or small dielectric constant. Reprinted with permission from R. E. Newnham, V. Sundar, R. Yimnirun, J. Su, and Q. M. Zhang, J. Phys. Chem. B 101, 10141 (1997). Copyright 1997 American Chemical Society. ${ }^{11}$

effects. As an electric field is applied, the cations and anions in a crystal structure are displaced in opposite directions by $\Delta r$. This displacement is responsible for the electric polarization, dielectric permittivity, and the electrostrictive strain. To the first approximation, ${ }^{1,11}$ the electrostrictive coefficient is written as

$$
Q=\frac{x}{P^{2}} \sim \frac{\Delta r}{(\Delta r)^{2}}=\frac{1}{\Delta r} \sim \frac{1}{\varepsilon},
$$

where $\varepsilon$ is the dielectric permittivity, $x$ is the strain, and $P$ is the polarization. For a certain electric field, a larger $\Delta r$ corresponds to a larger $\varepsilon$.

The electrostrictive coefficients were also closely associated with the elastic compliance $s$. Through the converse effect, electrostriction is proportional to the change in the inverse dielectric permittivity $\chi$ with stress $X$, which is expressed as

$$
Q \sim \frac{\Delta \chi}{X} \sim \frac{x}{(x / s)}=s,
$$

where $Q$ is the electrostrictive coefficient, $\Delta \chi$ is the variation of $\chi$ on the stress $X$, and $x$ is the strain on the stress $X$. To the first approximation, $\Delta \chi$ is proportional to the relative variations in the lattice parameters.

Combining Eqs. (22) and (23), the electrostrictive coefficient $Q$ is thought to be proportional to $s / \varepsilon$. For materials with a similar elasticity, the dielectric permittivity can be used to evaluate the level of the electrostrictive effect. Figure 13 illustrates $Q_{h}$ as a function of the dielectric constant for perovskite materials, where $\log \left(Q_{h}\right)$ follows a linear relationship against $\log (\varepsilon)$, as the elastic constants are of the same order for these materials.

For ferroelectric-related materials, the Curie-Weiss constant $C$ can be used to evaluate the level of the electrostrictive coefficients, because the dielectric permittivity is sensitive to temperature and phase transition points. According to thermodynamic analysis, the product of the electrostrictive

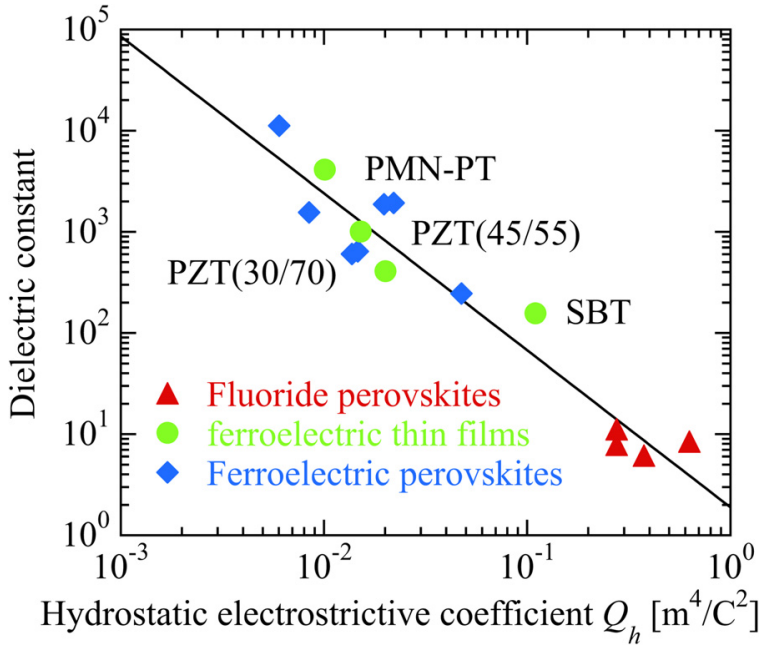

FIG. 13. Hydrostatic electrostrictive coefficient $Q_{h}$ as a function of the dielectric constant in perovskite materials. Reprinted with permission from R. E. Newnham, V. Sundar, R. Yimnirun, J. Su, and Q. M. Zhang, J. Phys. Chem. B 101, 10141 (1997). Copyright 1997 American Chemical Society. ${ }^{11}$

coefficient $Q_{h}$ and the Curie-Weiss constant $C$ was constant for all perovskite ferroelectrics. ${ }^{3}$ Table III gives the experimental data for $Q_{h} C$, including ferroelectrics, relaxors, and antiferroelectrics, where $Q_{h} C$ was $(3.1 \pm 0.5) \times 10^{3} \mathrm{~m}^{4} \mathrm{~K} / \mathrm{C}^{2}$ for perovskite ferroelectric materials. $2,7,37,51,63-68$

\section{Thermal expansion versus the electrostrictive effect}

The electrostriction and thermal expansion originate from similar mechanisms (i.e., potential anharmonicity), as such the thermal expansion and electrostrictive coefficients are thought to be interrelated. ${ }^{3}$ Based on the similar manner in deducing the electrostrictive coefficients (Sec. III), the thermal expansion can be written as ${ }^{47}$

$$
\alpha=\frac{3 g k_{B}}{4 f^{2} r_{0}}
$$

where $k_{B}$ is Boltzmann's constant and $r_{0}$ is the distance between a cation and its nearest-neighbor anion at equilibrium. $f$ and $g$ are the harmonic and anharmonic components, respectively. Similar to electrostriction, the thermal expansion coefficient was zero when the anharmonic effect was ignored $(g=0)$.

The thermal expansion coefficient $\alpha$ against the hydrostatic electrostrictive coefficient $Q_{h}$ for various dielectrics with cubic symmetry is plotted in Fig. 14, based on the reported results. ${ }^{3}$ From this figure, the empirical relation can be written as $\alpha=4.2 \times 10^{-5} Q_{h}^{0.5}$, which can be used to evaluate the electrostrictive effect from the thermal expansion coefficient.

In this section, the relationships between electrostriction and the macroscopic characteristics were reviewed for dielectric materials. Although the relationships could be used to evaluate the electrostrictive effect in dielectrics, they did not give accurate results. Some important factors were not considered, such as the crystal anisotropy, boundary conditions, and phase transitions. For ferroelectric materials, the effects of the domain walls, domain configurations, ${ }^{69-73}$ and 


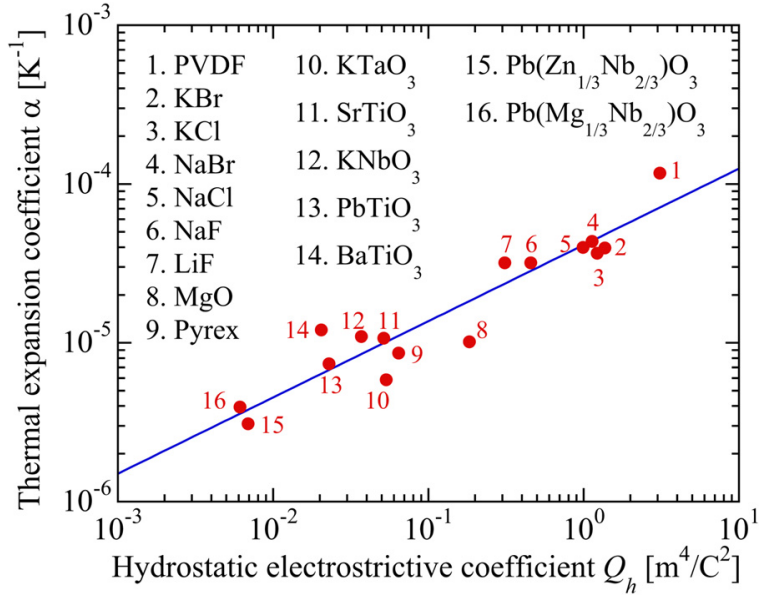

FIG. 14. Hydrostatic electrostrictive coefficient $Q_{h}$ with respect to thermal expansion coefficient for various dielectrics with cubic symmetry. Reprinted with permission from K. Uchino, S. Nomura, L. E. Cross, R. E. Newnham, and S. J. Jang, J. Mater. Sci. 16, 569 (1981). Copyright 1981 Springer. $^{3}$

the poling conditions ${ }^{74}$ on the electrostriction should also be considered.

\section{ELECTROSTRICTION IN PEROVSKITE FERROELECTRICS}

Extensive investigations on the electromechanical properties of ferroelectric single crystals, ceramics, and thin films have been a focus because their electric field-induced strains and piezoelectricity are much higher in comparison with other non-ferroelectric counterparts. In this section, the electrostrictive effects of perovskite ferroelectrics are discussed.

The reported electrostrictive coefficients for various perovskite ferroelectrics are listed in Table IV, ${ }^{3,75-84}$ including the data for single crystals and ceramics. The properties of single crystals are very important for materials exploration, as they allow for easy interpretation of the polycrystalline ceramic properties. On the other hand, some crystals are difficult to be grown; their ceramic counterparts can be used to evaluate the single crystal properties. The difference in the electrostrictive effect between single crystals and polycrystalline ceramics is discussed here.

For perovskite ferroelectric materials, the longitudinal electrostrictive coefficients $Q_{33}$ of the polycrystalline ceramics and thin films are generally much smaller than those of $\langle 100\rangle$ oriented single crystals. This phenomenon can be attributed to the following two factors. First, the strong anisotropy of electrostriction presents in perovskite crystals, which will be discussed in Sec. VC. Second, it is difficult to eliminate the contribution of the domain wall motion in polycrystalline ceramics during the electrostriction measurements. As a result, the measured data for the polycrystalline materials include contributions from the domain wall motion and grain orientation. In addition, for ferroelectric thin films, the values of the electrostrictive coefficients were expected to be further affected by substrate clamping, which generally decreased the $Q$ coefficients. ${ }^{85}$ It should be noted that when compared with the electrostrictive coefficient $Q_{33}$, the hydrostatic coefficient $Q_{h}$ was independent of the orientation for perovskite ferroelectric materials. ${ }^{86}$ Meanwhile, $Q_{h}$ was generally determined by measuring the inverse of the dielectric permittivity under hydrostatic pressure, where the influence of the domain wall was quite small when compared with the high field measurements. Thus, the hydrostatic electrostrictive coefficient $Q_{h}$ of the polycrystalline ceramics was found to be the same to that of the single crystals.

TABLE IV. The electrostrictive coefficients for perovskite crystals and ceramics; the coordinate system for crystals is the standard coordinate of cubic phase (X: [100], Y: [010], Z: [001]).

\begin{tabular}{|c|c|c|c|c|c|}
\hline \multirow[b]{2}{*}{ Crystals } & \multicolumn{4}{|c|}{ Electrostrictive coefficients $\left(\mathrm{m}^{4} / \mathrm{C}^{2}\right)$} & \multirow[b]{2}{*}{ References } \\
\hline & $Q_{11}^{\mathrm{C}}=Q_{1111}^{\mathrm{C}}$ & $Q_{12}^{\mathrm{C}}=Q_{1122}^{\mathrm{C}}$ & $Q_{44}^{\mathrm{C}}=2 Q_{1212}^{\mathrm{C}}$ & $\frac{Q_{11}^{\mathrm{C}}-Q_{12}^{\mathrm{C}}}{Q_{44}^{\mathrm{C}}}$ & \\
\hline PMN-0.28PT & 0.055 & -0.024 & 0.021 & 3.8 & 26 \\
\hline $\mathrm{PMN}-0.32 \mathrm{PT}$ & 0.056 & -0.022 & 0.019 & 4.1 & 26 \\
\hline PMN-0.37PT & 0.056 & -0.024 & 0.018 & 4.4 & 26 \\
\hline $\mathrm{PbTiO}_{3}$ & 0.089 & -0.030 & 0.034 & 3.2 & 51 \\
\hline $\mathrm{BaTiO}_{3}$ & 0.110 & -0.045 & 0.059 & 2.6 & 37 \\
\hline $\mathrm{Pb}\left(\mathrm{Yb}_{1 / 2} \mathrm{Nb}_{1 / 2}\right) \mathrm{O}_{3}$ & 0.082 & & & & 77 \\
\hline $\mathrm{Pb}\left(\mathrm{Zn}_{1 / 3} \mathrm{Nb}_{2 / 3}\right) \mathrm{O}_{3}$ & 0.063 & -0.25 & 0.24 & 3.7 & This work \\
\hline $\mathrm{Pb}\left(\mathrm{Mg}_{1 / 3} \mathrm{Nb}_{2 / 3}\right) \mathrm{O}_{3}$ & 0.027 & -0.01 & 0.01 & 3.7 & 3 and 84 \\
\hline$\left(\mathrm{Bi}_{1 / 2} \mathrm{Na}_{1 / 2}\right)_{1-\mathrm{x}} \mathrm{Ba}_{\mathrm{x}} \mathrm{Zr}_{\mathrm{y}} \mathrm{Ti}_{1-\mathrm{y}} \mathrm{O}_{3}$ & $0.023-0.04$ & & & & 78 \\
\hline \multicolumn{6}{|l|}{ Ceramics } \\
\hline $\mathrm{PbZr}_{0.52} \mathrm{Ti}_{0.48} \mathrm{O}_{3}$ & & $-0.007 \sim-0.016$ & & & 75 \\
\hline $\begin{array}{l}\mathrm{La} \text { doped } 0.65 \mathrm{PbZrO}_{3}-0.35 \mathrm{PbTiO}_{3} \\
\text { (molar percentage of } \mathrm{La} \text { is } 7 \%-10 \% \text { ) }\end{array}$ & $0.018-0.022$ & $-0.008 \sim-0.0012$ & & & 76 \\
\hline $\mathrm{PMN}-0.1 \mathrm{PT}$ & l & -0.009 & & & 4 \\
\hline $\mathrm{Bi}_{0.5} \mathrm{Na}_{0.5} \mathrm{TiO}_{3}-\mathrm{K}_{0.5} \mathrm{Na}_{0.5} \mathrm{NbO}_{3}$ & $0.015-0.018$ & & & & 80 \\
\hline $\mathrm{Bi}_{0.5} \mathrm{Na}_{0.5} \mathrm{TiO}_{3}-\mathrm{BaTiO}_{3}-\mathrm{K}_{0.5} \mathrm{Na}_{0.5} \mathrm{NbO}_{3}$ & $0.021-0.026$ & & & & 81 \\
\hline $\mathrm{Bi}_{0.5} \mathrm{Na}_{0.5} \mathrm{TiO}_{3}-\mathrm{BaTiO}_{3}$ & 0.022 & & & & 82 \\
\hline $\mathrm{Bi}_{1 / 2}\left(\mathrm{Na}_{0.82} \mathrm{~K}_{0.18}\right)_{1 / 2} \mathrm{TiO}_{3}$ & 0.023 & & & & 83 \\
\hline $\mathrm{K}_{0.5} \mathrm{Na}_{0.5} \mathrm{NbO}_{3}-\mathrm{SrTiO}_{3}$ & $0.004-0.007$ & & & & 79 \\
\hline
\end{tabular}




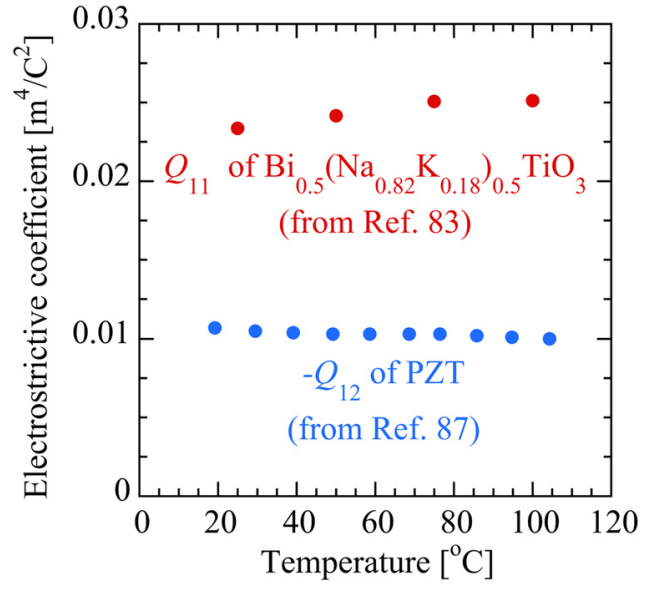

FIG. 15. The temperature dependence of electrostrictive coefficient $Q$ of $\mathrm{Bi}_{0.5}\left(\mathrm{Na}_{0.82} \mathrm{~K}_{0.18}\right)_{0.5} \mathrm{TiO}_{3}$ and PZT ceramics. After Refs. 83 and 87.

In the following sections, the effects of the ferroelectric phase transition and crystal orientation on electrostrictive coefficients $Q$ are evaluated. The electrostrictive coefficients $Q$ are discussed for perovskite ferroelectrics with respect to electromechanical properties, such as the electric fieldinduced strain and piezoelectric coefficients, in order to find alternative ways to enhance the piezoelectric activity.

\section{A. Electrostrictive effect versus ferroelectric phase transitions}

The phase transition point in perovskite ferroelectrics, around where the dielectric and piezoelectric responses generally show peak values, has been extensively studied over the last 70 years. In this section, the electrostrictive coefficients $Q$ of perovskite ferroelectrics are discussed with respect to the phase transition points (induced by temperature or composition).

\section{Polymorphic phase transition (PPT, phase transitions induced by temperature)}

For typical ferroelectrics, such as $\mathrm{BaTiO}_{3}, \mathrm{PbTiO}_{3}$, and $\mathrm{LiNbO}_{3}$, the electrostrictive coefficients $Q$ are temperature-independent. This notation is generally accepted based on thermodynamic analysis. ${ }^{51}$ Meanwhile, the $Q$ coefficients calculated from first principals at $0 \mathrm{~K}$ were consistent with the results measured at room temperature, ${ }^{44,45}$ again revealing that the effect of temperature on the $Q$ coefficients is minimal for classical ferroelectrics. From the experimental aspect, the temperature-independent electrostrictive coefficients $Q$ were also observed in PZT and $\mathrm{Bi}_{0.5}\left(\mathrm{Na}_{0.82} \mathrm{~K}_{0.18}\right)_{0.5} \mathrm{TiO}_{3}$ ferroelectric systems, as shown in Fig. $15^{83,87}$

To understand the temperature dependence of the electrostrictive behavior in relaxor/relaxor-PT single crystals, related parameters, including the piezoelectric coefficient, dielectric constant, spontaneous polarization, and electrostrictive coefficients, as a function of the temperature were investigated for single-domain PMN- $x$ PT crystals, as shown in Fig. 16. The large variations in the dielectric and piezoelectric coefficients are closely associated with the PPT, where the increase in the dielectric and piezoelectric properties for PMN-0.28PT and PMN-0.32PT crystals with increasing temperature was attributed to the crystals approaching a PPT $\left(T_{\mathrm{R}-\mathrm{T}}\right.$ and $T_{\mathrm{O}-\mathrm{T}}$ are $95^{\circ} \mathrm{C}$ and $80^{\circ} \mathrm{C}$, respectively), while the decrease in the dielectric and piezoelectric responses for PMN-0.37PT occurred because the PPT of PMN-0.37PT was below room temperature $\left(\sim-45^{\circ} \mathrm{C}\right) .^{21}$ The spontaneous polarization decreased with increasing temperature, caused by the pyroelectric effect. ${ }^{88}$ Of particular significance is that the electrostrictive coefficients of the PMN- $x$ PT crystals were insensitive to temperature and PPT, regardless of the strong temperature dependence of the piezoelectric/dielectric properties. The temperature-independent electrostrictive coefficients $Q_{11}$ and $Q_{12}$ were also observed in PMN crystals, as shown in Fig. 17.

It should be noted that the reported data for the PMN, PMN-PT, and $\mathrm{Bi}_{0.5}\left(\mathrm{Na}_{0.82} \mathrm{~K}_{0.18}\right)_{0.5} \mathrm{TiO}_{3}$ systems were below the $T_{C}$. At temperatures above $T_{C}$ (or above the Burns temperature $T_{B}$ for relaxor ferroelectrics; for PMN and PMN-PT crystals, $T_{B} \sim 300{ }^{\circ} \mathrm{C}^{29}$ ), the electrostrictive coefficient $Q_{11}$ was found to decrease linearly with increasing temperature for $\mathrm{BaTiO}_{3}$ and tin modified lead zirconate (PZS) crystals (Fig. 18), ${ }^{89,90}$ which can be explained using the phenomenological theory. ${ }^{91}$
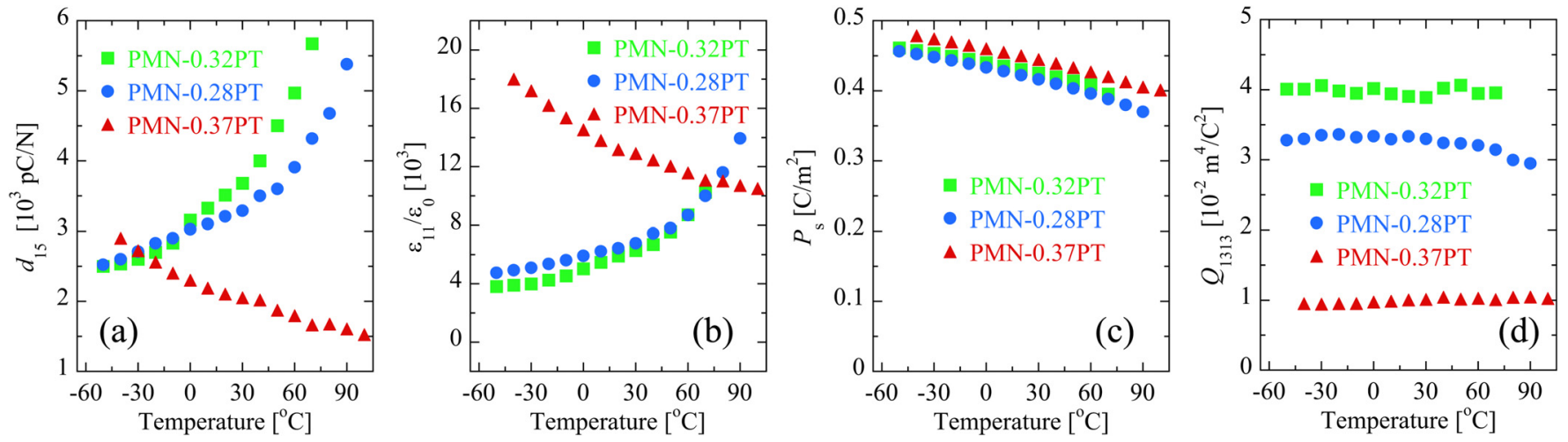

FIG. 16. The temperature-dependent properties, (a) piezoelectric coefficient $d_{15}$, (b) dielectric constant $\varepsilon_{11}$, (c) spontaneous polarization $P_{s}$, and (d) electrostrictive coefficient $Q_{1313}$ for single domain PMN-0.28PT, PMN-0.32PT, and PMN-0.37PT crystals. The coefficients of rhombohedral, orthorhombic, and tetragonal crystals were measured in the standard coordinates of $3 \mathrm{~m}, \mathrm{~mm} 2$, and $4 \mathrm{~mm}$ symmetries, respectively. The PPTs of PMN-0.28PT, PMN-0.32PT, and PMN-0.37PT crystals are 93, 80, and $-45^{\circ} \mathrm{C}$, respectively. Reprinted with permission from Appl. Phys. Lett. 102, 152910 (2013). Copyright 2013 AIP Publishing LLC. ${ }^{26}$ 


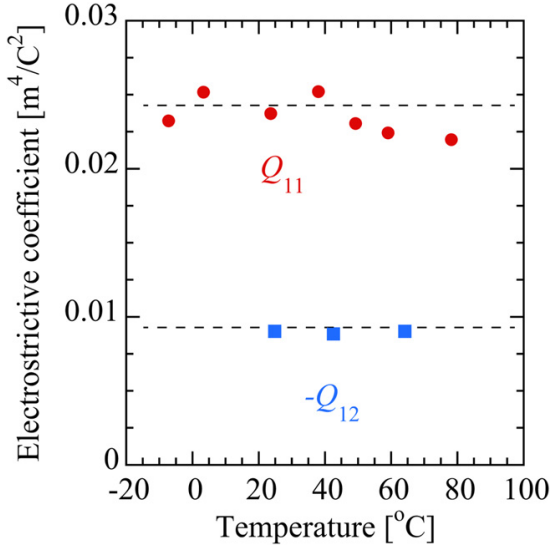

FIG. 17. The temperature dependence of electrostrictive coefficient $Q$ of PMN crystals. Reprinted with permission from J. Appl. Phys. 51, 1142 (1980). Copyright 1980 AIP Publishing LLC. ${ }^{2}$

\section{Morphotropic phase boundary (MPB, phase transitions induced by composition)}

The MPB is an important characteristic for many perovskite ferroelectric solid solutions as it accounts for the enhanced dielectric, elastic, and piezoelectric properties. In this section, the effect of MPB on electrostriction is surveyed for perovskite ferroelectrics.

As listed in Table IV, the $Q$ coefficients were found to have similar values for compositions around the MPBs in PMN-PT and $\mathrm{Bi}_{0.5} \mathrm{Na}_{0.5} \mathrm{TiO}_{3}-\mathrm{BaTiO}_{3}-\mathrm{K}_{0.5} \mathrm{Na}_{0.5} \mathrm{NbO}_{3}$ systems. However, Fig. 19 outlines the $Q$ coefficients for the PZ- $x$ PT crystals, exhibiting a slight increase around the MPB composition. ${ }^{51}$ This was thought to be associated with the domain wall motion or phase transition during the electrostrictive measurements, because the $Q$ values for the PZT crystals were inferred from the measurements on the polycrystalline ceramics.

The electrostrictive coefficients $\left(Q_{11}^{\mathrm{C}}-Q_{12}^{\mathrm{C}}\right)^{92}$ of [001] poled PMN- $x$ PT and PZN- $x$ PT crystals are derived from the data in Refs. 2, 18, 52, and 93-97 and plotted in Fig. 20. The electrostrictive coefficients of the relaxor-PT crystals generally increase with increasing PT content, with no abnormal phenomenon observed around the MPB. Enhancement of the electrostrictive coefficient $\left(Q_{11}^{\mathrm{C}}-Q_{12}^{\mathrm{C}}\right)$ with increasing $\mathrm{PT}$ content was attributed to the enhancement in the ordering degree of the relaxor-PT crystals by increasing the PT

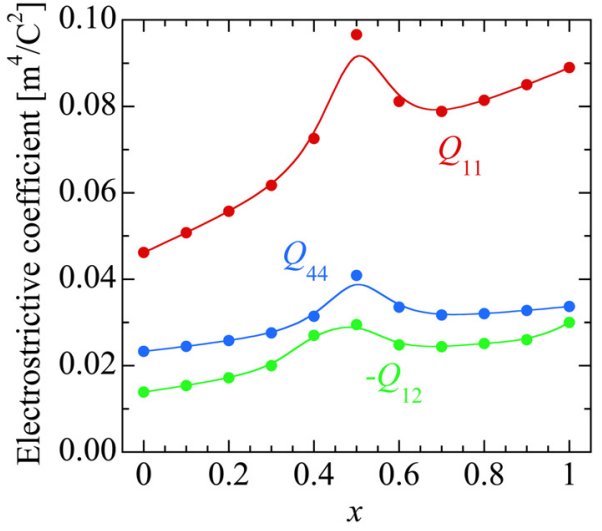

FIG. 19. The composition dependence of $Q$ coefficients for PZ- $x$ PT crystals, where $x$ represents the PT concentration.

content, as discussed in Sec. IV. Vikhnin et al. theoretically explained the increase in the electrostrictive coefficient $Q$ as a function of the PT content by considering the quasi-local Ti-O stretching mode in PMN-PT solid solutions. ${ }^{61}$ It should be noted that for PZN- $x$ PT crystals with $x=0.08$ and 0.12 , the electrostriction was comparable to that of the PT crystals, while the electrostrictive coefficients $\left(Q_{11}^{\mathrm{C}}-Q_{12}^{\mathrm{C}}\right)$ for the PMN- $x$ PT crystals were much lower than those of PT crystals, even in the composition range of $x=0.26-0.42$. This result revealed that the $\mathrm{B}$-site ordering degree for the PZN- $x$ PT crystals was higher than that for the PMN- $x$ PT crystals at the MPB composition.

Overall, the influences of the PPT and MPB on the $Q$ electrostrictive coefficients were minimal in comparison with the effects on the dielectric and piezoelectric properties in perovskite ferroelectrics. This demonstrates that the magnitude of the electrostrictive coefficient does not strongly depend on various phases (ferroelectric, antiferroelectric, or non-polar phases) and phase transitions. For perovskite ferroelectrics, the phase transition (ferroelectric, antiferroelectric, paraelectric) is accompanied by atomic displacements that are dominated by motion of the B cations in the oxygen octahedron network. ${ }^{32}$ From the respect of crystal structure, the difference between the paraelectric, antiferroelectric, and various ferroelectric phases is reflected in the displacement of ions, including the direction and magnitude of the displacement. However, it is important to note that the
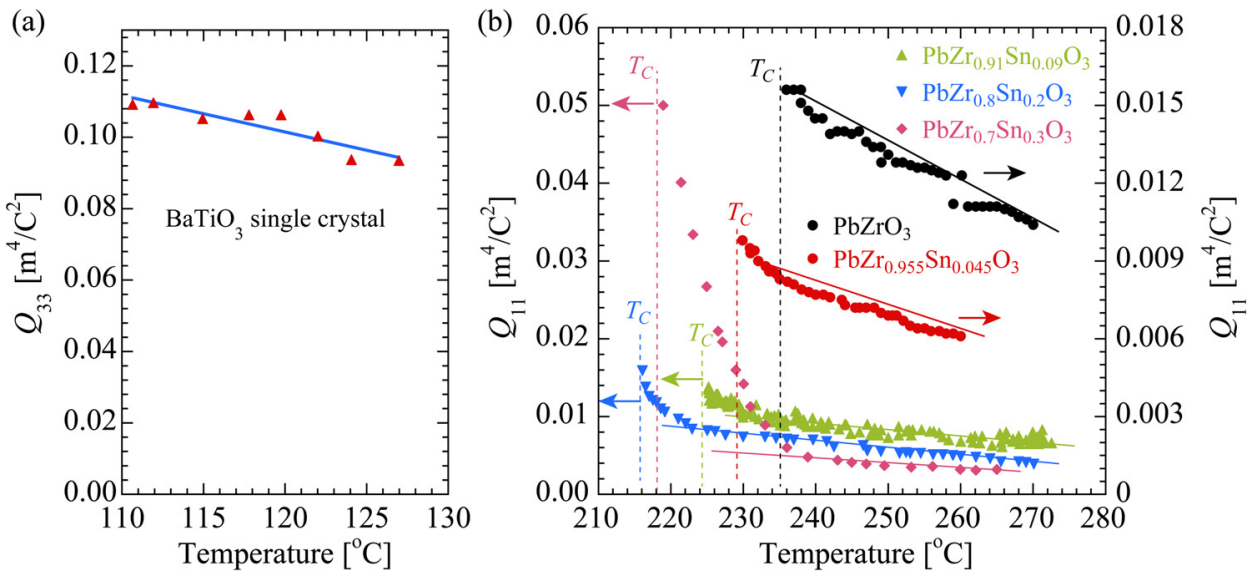

FIG. 18. The temperature dependence of electrostrictive coefficient $Q$ for (a) BT and (b) PZ and PZS single crystals at the temperature above their respective $T_{C} \mathrm{~s}$. (a) Reprinted with permission from W. Pan, Q, Zhang, A. S. Bhalla, and L. E. Cross, J. Am. Ceram. Soc. 71, C-302 (1998). Copyright 2005 John Wiley and Sons. ${ }^{90}$ (b) Reprinted with permission from I. JankowskaSumara, K. Roleder, A. Majchrowski, and J. Zmija, J. Adv. Dielectr. 1, 223 (2011). Copyright 2011 World Scientific Publishing Company. ${ }^{89}$ 


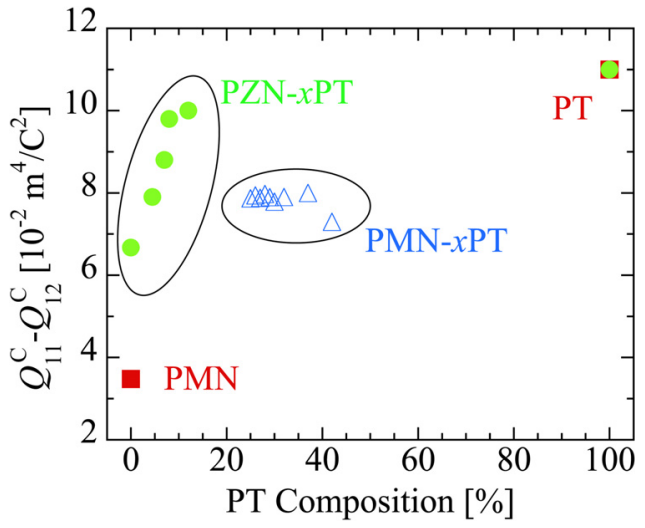

FIG. 20. Composition dependence of electrostrictive coefficient for relaxorPT crystals. The data of PMN, PZN, PZN-0.045PT, PZN-0.07PT, PZN0.08PT, PZN-0.12PT, PMN-0.42PT, and PT crystals are from Refs. 2, 18, 51,87-91.

displacements are relatively small in perovskite structures, being $<5 \%$ of the lattice parameter, revealing that the ionpair potential energy and lattice parameters [the parameters in Eq. (18)] were not greatly affected by these small displacements. As a result, electrostriction in perovskite crystals is not sensitive to the ferroelectric phases and phase transitions.

\section{B. Orientation dependence of electrostriction}

The orientation dependence of the electrostrictive coefficients $Q_{33}^{*}{ }^{98}$ for BT and PMN-0.28PT crystals were calculated by the axis transformation using the data in Table IV, as shown in Fig. 21. The maximum electrostrictive coefficient $Q_{33}^{*}$ was along the $\langle 100\rangle$ direction, while the moderate and minimum values were along the $\langle 110\rangle$ and $\langle 111\rangle$ directions, respectively. Similar anisotropic behavior was observed in PMN-0.32PT, PMN-0.37PT, and other perovskite crystals, due to the fact that the anisotropic factors $\left(Q_{11}^{\mathrm{C}}-Q_{12}^{\mathrm{C}}\right) / Q_{44}^{\mathrm{C}}$ is much larger than 1 for those crystals, as listed in Table IV ( $Q_{33}^{*}$ will be an isotropic coefficient if $\left.\left(Q_{11}^{\mathrm{C}}-Q_{12}^{\mathrm{C}}\right) / Q_{44}^{\mathrm{C}}=1\right)$. Similar anisotropic behavior was also reported in other ionic crystals possessing oxygen octahedral structures $\left(\mathrm{LiNbO}_{3}, \quad \mathrm{LiTaO}_{3}, \mathrm{KNbO}_{3}, \quad\right.$ and $\mathrm{Ba}_{2} \mathrm{NaNb}_{5} \mathrm{O}_{15}$ crystals), as listed in Table V. ${ }^{54}$ As demonstrated in Ref. 53, the anisotropy of the electrostriction in perovskite crystals led us to believe that it is inherently associated with the oxygen octahedra.

Figure 22 shows the variation in the oxygen octahedral structure as polarization appeared along different crystallographic directions. When a $\mathrm{B}$-site cation moves along the [001] direction, an oxygen anion $\mathrm{O}_{1}$ will be "pushed" upward to release the energy that originated from compressing the distance of B-O ${ }_{1}$, as shown in Fig. 22(b). Consequently, the lattice parameter along the [001] direction is elongated by $d$. The strain along the [001] direction can be expressed as $d / a$. Figure 22(c) shows the scenario where a B-site cation moves along the [110] direction. In this case, the oxygen $\mathrm{O}_{3}$ and $\mathrm{O}_{4}$ anions will move along the [110] or $[1 \overline{1} 0] /[\overline{1} 10]$ directions to release the $\mathrm{B}-\mathrm{O}_{3}$ and $\mathrm{B}-\mathrm{O}_{4}$ pair potential because the direction of B-O bond $(\langle 100\rangle)$ is not parallel to the [110] direction. It should be noted that the electrostrictive coefficient $Q_{33}^{*}$ along the [110] direction is reduced by the motion of the $\mathrm{O}_{3} / \mathrm{O}_{4}$ ions along the $[1 \overline{1} 0] /[\overline{1} 10]$ directions, because the $[1 \overline{1} 0] /[\overline{1} 10]$
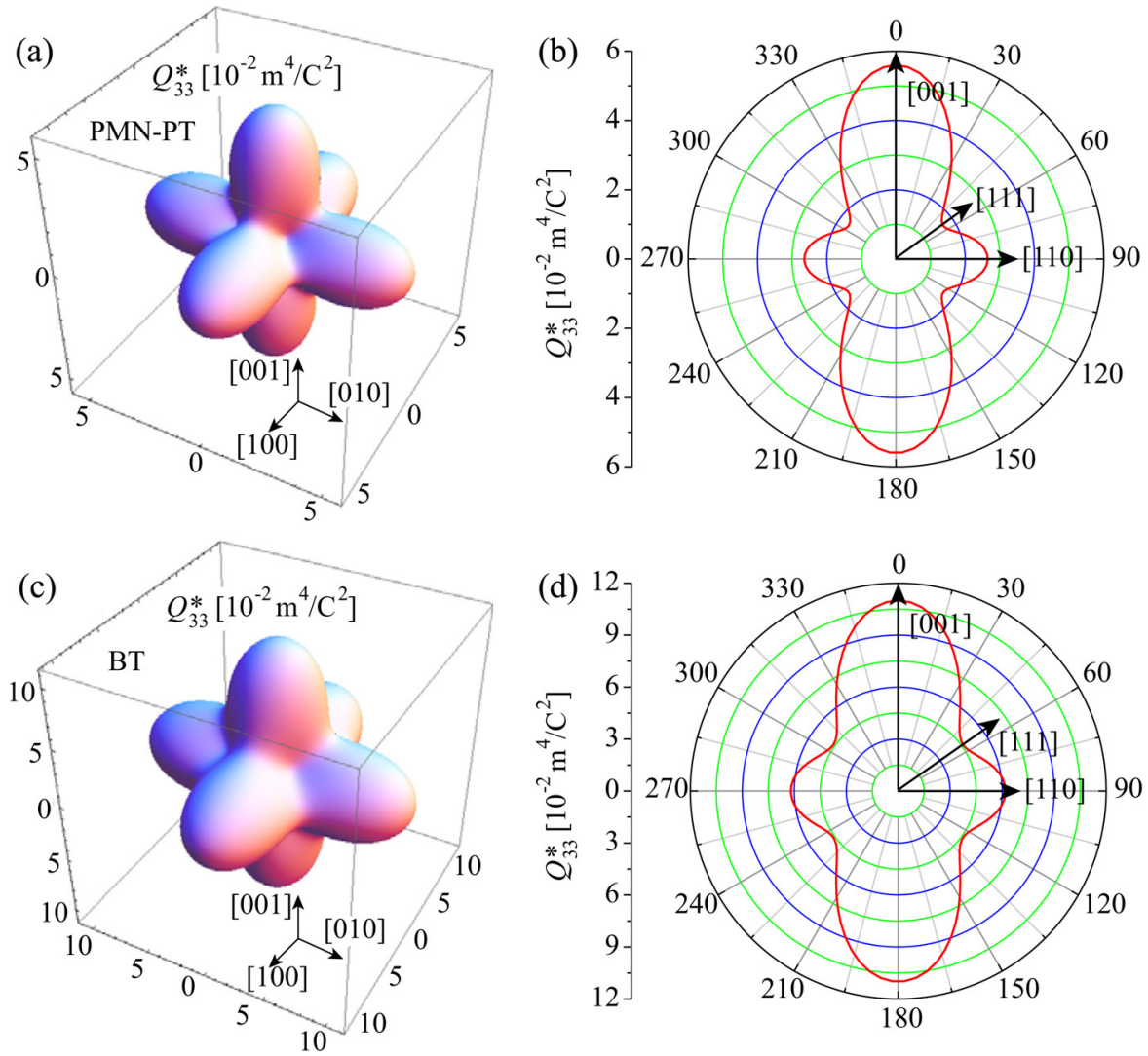

FIG. 21. The orientation dependence of electrostrictive coefficient $Q_{33}^{*}$ for BT and PMN-0.28PT crystals. (a) Three-dimensional figure for PMN$0.28 \mathrm{PT}$, (b) cross section of $(1 \overline{1} 0)$ plane for PMN-0.28PT, (c) threedimensional figure for BT crystal, (d) cross section of $(1 \overline{1} 0)$ plane. 
TABLE V. $Q^{\mathrm{p}}$ are electrostrictive coefficients normalized to those of the perovskite-oxide structure. Data are from Ref. 53.

\begin{tabular}{lcccc}
\hline \hline & \multicolumn{4}{c}{ Electrostrictive coefficients $\left(\mathrm{m}^{4} / \mathrm{C}^{2}\right)$} \\
\cline { 2 - 5 } Crystals & $Q_{11}^{\mathrm{p}}$ & $Q_{12}^{\mathrm{P}}$ & $Q_{44}^{\mathrm{P}}$ & $\frac{Q_{11}^{\mathrm{C}}-Q_{12}^{\mathrm{C}}}{Q_{44}^{\mathrm{C}}}$ \\
\hline $\mathrm{Ba}_{2} \mathrm{NaNb}_{5} \mathrm{O}_{15}$ & 0.11 & -0.019 & 0.029 & 4.4 \\
$\mathrm{LiNbO}_{3}$ & 0.096 & -0.039 & 0.024 & 5.6 \\
$\mathrm{LiTaO}_{3}$ & 0.085 & -0.033 & 0.033 & 3.6 \\
\hline \hline
\end{tabular}

directions are perpendicular to the [110] direction. Analogous to the [110] direction, a similar scenario occurs for the [111] direction, as shown in Fig. 22(d). As a B-site cation moves along the [111] direction, the oxygen ions can move along the directions perpendicular to [111] direction to release the B-O pair potential, which mitigates the $Q_{33}^{*}$ along the [111] direction. The difference between the [111] and [110] directions is that in the former, the oxygen ions are easier to move along the perpendicular direction to release the compression energy. This is because the angle between the [111] and B-O chain directions is $54^{\circ}$, which is larger than the angle between the [110] and $\mathrm{B}-\mathrm{O}$ bond directions $\left(45^{\circ}\right)$. Based on this approach, electrostrictive anisotropy of perovskite crystals has been explained qualitatively, which is related to the oxygen octahedral structure. The maximum electrostrictive coefficient $Q_{33}^{*}$ was along the $\langle 100\rangle$ direction because the B-O chains align along the $\langle 100\rangle$ direction. Using the same approach, the electrostrictive anisotropy for specific crystals can be explained. For example, for fluoride perovskite crystals, the maximum longitudinal electrostrictive coefficient $Q_{33}^{*}$ is along the $\langle 100\rangle$ directions, as the B-F chains align along this direction; while maximum $Q_{33}^{*}$ is along the $\langle 111\rangle$ directions for the fluorite crystal $\mathrm{CaF}_{2}$, as the

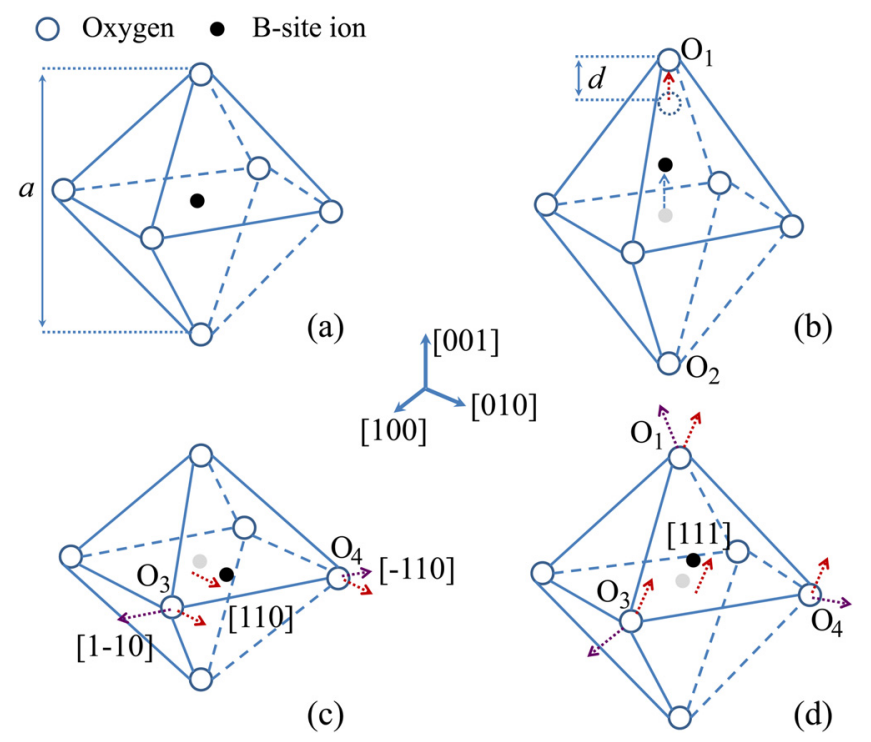

FIG. 22. The deformation of oxygen-octahedral structure with different polarization directions. (a) Zero polarization; (b) polarization along [001] direction; (c) polarization along [110] direction; (d) polarization along [111] direction. The red arrows for oxygen ions indicate that the oxygen ions move along the polarization direction. The purple arrows for oxygen ions indicate that oxygen ions move along the direction perpendicular to the polarization direction.
Ca-F chains align along the $\langle 111\rangle$ directions, as shown in Fig. 23.

In addition to the longitudinal electrostrictive coefficient $Q_{33}^{*}$, the other electrostrictive coefficients for perovskite crystals also showed strong anisotropic behavior. Two examples are: (a) In the coordinate system shown in Fig. 24(a), the $Q_{32}^{*}$ coefficient was positive, indicating that the extensions occurred simultaneously parallel and perpendicular to the applied electric field ${ }^{86}$ and (b) the maximum shear coefficient for the perovskite crystal is presented in the coordinate shown in Fig. 24(b), being equal to $Q_{1313}^{*}=\frac{1}{2}\left(Q_{11}^{C}-Q_{12}^{C}\right)$.

\section{Electrostrictive coefficient $Q$ versus the electromechanical properties}

\section{Electrostrictive coefficient $Q$ versus the electric field-induced strain}

In general, an electric field-induced strain can be written as

$$
S=g \cdot P(E)+Q \cdot P^{2}(E)+\cdots,
$$

where the first and second terms represent the piezoelectric and electrostrictive strains, respectively, $P(E)$ is the electric field-induced polarization and $g$ is the piezoelectric voltage coefficient. The strains related to the high order of $P$ were ignored in Eq. (25). For piezoelectric crystals without ferroelectricity, the piezoelectric strain is usually much higher than the electrostrictive strain, such as in quartz crystals. On the contrary, based on the phenomenological theory, the strain in ferroelectrics with centrosymmetric parent phases is induced by the electrostriction only. ${ }^{32}$ These ferroelectrics include $\mathrm{LiNbO}_{3}, \mathrm{BaTiO}_{3}, \mathrm{PbTiO}_{3}, \mathrm{KNbO}_{3}$, and other related materials. Thus, Eq. (25) can be expressed as Eq. (26) for these materials

$$
S=Q P^{2}(E) \sim Q \varepsilon^{2} E^{2}
$$

where $\varepsilon$ is the dielectric permittivity. In Eq. (26), the anisotropy and nonlinear properties for the dielectric response were not considered. Based on Eq. (26), the figure of merit used to evaluate the level of strain induced by a certain electric field is $Q \varepsilon^{2}$. It was observed that a large $Q$ coefficient does not necessarily give rise to large electric field-induced strains, because a high $Q$ coefficient generally corresponds to a low dielectric permittivity, as discussed in Sec IV B 1 $(Q \propto 1 / \varepsilon)$. It was reported that the $Q$ coefficient in the relaxor ferroelectric PMN was about one fifth that of a typical ferroelectric BT, while the dielectric permittivity of PMN was 5-10 times higher than that in BT. Thus, PMN exhibited a superior electric field-induced strain when compared with $\mathrm{BaTiO}_{3}$. In addition, perovskite materials generally showed a larger electric field-induced strain because the dielectric permittivity for perovskites is significantly higher than other ionic crystals.

It should be noted that domain switching can also be used to enhance the electric field-induced strain in ferroelectric crystals. Interesting results were reported for [001] oriented BT crystals with Mn dopants or preloaded stress in 


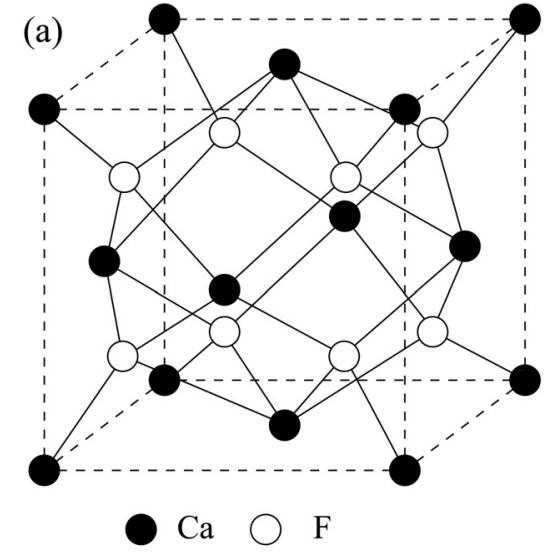

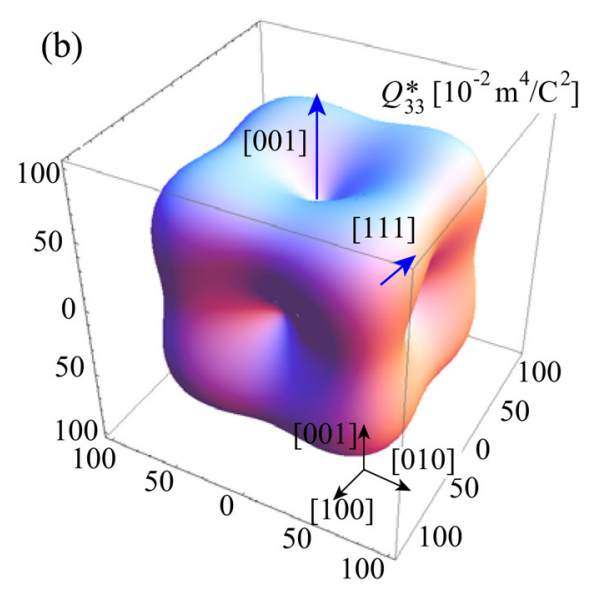

FIG. 23. The relationship of crystal structure and orientation dependent electrostriction for fluoride crystal. (a) The structure of fluoride crystal; (b) the orientation dependence of $Q_{33}^{*}$ for $\mathrm{CaF}_{2}$. Input data are from Ref. 11 . which $\sim 1 \%$ strain was induced with an electric field. ${ }^{99-101}$ With a decrease in the applied electric field, Mn dopants or preloaded stress made the domains switch back to the directions that were perpendicular to the applied electric field, as shown in Fig. 25. Theoretically, the maximum strain induced by domain switching in [001] oriented tetragonal crystals can be expressed as $S_{3}=\left(Q_{11}-Q_{12}\right) P_{s}^{2}$ (as depicted in Fig. 25), which is much larger than that induced by lattice deformation. Through domain switching, the level of maximum strain is only related to the electrostrictive coefficients and the spontaneous polarization and not to the dielectric permittivity. However, the strain induced by domain switching is generally accompanied by a large hysteresis, limiting its potential application in electromechanical devices.

\section{Electrostrictive coefficient $Q$ versus piezoelectric activity}

Analogous to the electric field-induced strain, the intrinsic piezoelectricity of ferroelectrics with centrosymmetry parent phases comes from the electrostrictive effect. Compared with the extrinsic contribution (domain wall motion), the electrostrictive contribution showed low hysteresis and high stability with respect to the frequency and electric field. ${ }^{69,70,102}$ In addition, the sign of the piezoelectric coefficient $d$ can also be determined by the sign of the electrostrictive coefficient $Q$. For example, the longitudinal piezoelectric coefficient $d_{33}$ has a positive value for ferroelectric crystals and ceramics, whereas $d_{33}$ has a negative value for ferroelectric polymers,
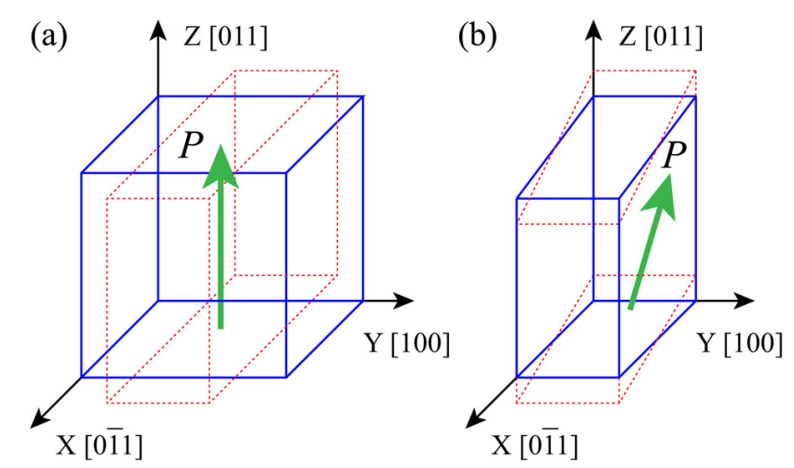

FIG. 24. Schematic of coordinate system and corresponding polarization and electrostrictive strains for perovskite crystals. (a) Positive $Q_{3322}^{*}$ coefficient; (b) maximum $Q_{1313}^{*}$ coefficient. because the electrostrictive coefficient was positive for ionic crystals and negative for polymers. Furthermore, the anisotropy of the electrostriction plays an important role in the piezoelectric anisotropy in ferroelectric crystals. To illustrate this scenario, the detailed relationship between the piezoelectric and electrostrictive coefficients are given for relaxor-PT crystals, whose parent phase and symmetry were cubic and $m \overline{3} m$, respectively.

According to Eq. (2) and the transformation of the coordinate system, the piezoelectric coefficient and dielectric permittivity for [001] poled rhombohedral crystals can be written as

$$
\varepsilon_{33}^{[001]}=\frac{1}{3}\left(\varepsilon_{33}^{\mathrm{R}}+2 \varepsilon_{11}^{\mathrm{R}}\right),
$$

$$
\begin{aligned}
d_{33}^{[001]} & =2 P_{s}^{\mathrm{C}}\left(\varepsilon_{33}^{\mathrm{C}} Q_{33}^{\mathrm{C}}+2 \varepsilon_{31}^{\mathrm{C}} Q_{31}^{\mathrm{C}}\right) \\
& =\frac{2 \sqrt{3}}{3} P_{s}^{\mathrm{R}}\left[\left(\frac{2}{3} \varepsilon_{11}^{\mathrm{R}}+\frac{1}{3} \varepsilon_{33}^{\mathrm{R}}\right) Q_{33}^{\mathrm{C}}+2\left(\frac{2}{3} \varepsilon_{33}^{\mathrm{R}}-\frac{1}{3} \varepsilon_{11}^{\mathrm{R}}\right) Q_{31}^{\mathrm{C}}\right]
\end{aligned}
$$

where the symbols $\mathrm{R}$ and $\mathrm{C}$ indicate the rhombohedral $3 m$ and cubic $m \overline{3} m$ coordinate systems in which the property coefficients were measured (see details in the Appendix). An important characteristic in the relaxor-PT crystals is that the transverse dielectric permittivity $\varepsilon_{11}$ is much higher than the

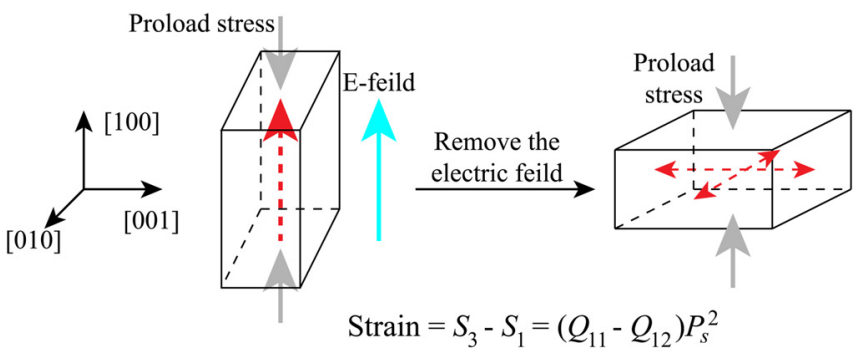

FIG. 25. Schematic for [001] oriented tetragonal BT crystal under electric field and preload stress. The dashed arrows represent the polarization directions. $P_{s}$ is the spontaneous polarization, $S_{1}$ and $S_{3}$ are the spontaneous strain along $a$ and $c$ axes of tetragonal BT crystal, respectively. As shown in this figure, domains are aligned along [001] direction with applying E-field of [001] direction, while the domains will switch back to the four possible directions as the E-field removed, where the polarizations are perpendicular to the preload stress ([001] direction). For Mn-doped BT crystals, similar domain switching will occur due to the internal bias field. 
longitudinal dielectric permittivity $\varepsilon_{33} .{ }^{20}$ Thus, Eqs. (27) and (28) can be simplified for relaxor-PT crystals as

$$
\begin{gathered}
\varepsilon_{33}^{[001]} \approx \frac{2}{3} \varepsilon_{11}^{\mathrm{R}}, \\
d_{33}^{[001]} \approx \frac{2 \sqrt{3}}{3} P_{s}^{\mathrm{R}} \varepsilon_{33}^{[001]}\left(Q_{33}^{\mathrm{C}}-Q_{31}^{\mathrm{C}}\right),
\end{gathered}
$$

where $\varepsilon_{33}^{[001]}$ is the longitudinal dielectric permittivity for [001] poled rhombohedral crystals, $\varepsilon_{11}^{R}$ is the transverse dielectric permittivity measured in the standard coordinate system for rhombohedral $3 m$ symmetry (see details in the Appendix), and $P_{S}^{\mathrm{R}}$ is the spontaneous polarization of the rhombohedral crystals. Based on the same approximation, the piezoelectric coefficient $d_{33}$ of [001] poled orthorhombic relaxor-PT crystals and [111] poled tetragonal relaxor-PT crystals can be written as

$$
\begin{aligned}
& d_{33}^{[001]} \approx \frac{2 \sqrt{2}}{2} P_{s}^{\mathrm{O}} \varepsilon_{33}^{[001]}\left(Q_{33}^{\mathrm{C}}-Q_{31}^{\mathrm{C}}\right), \\
& d_{33}^{[111]} \approx \frac{2 \sqrt{3}}{3} P_{s}^{\mathrm{T}} \varepsilon_{33}^{[111]}\left(Q_{33}^{\mathrm{R}}-Q_{31}^{\mathrm{R}}\right),
\end{aligned}
$$

where $Q_{33}^{\mathrm{R}}$ and $Q_{31}^{\mathrm{R}}$ are the electrostrictive coefficients measured in the standard coordinate system for a rhombohedral $3 m$ symmetry. ${ }^{92}$

As expressed in Eqs. (30)-(32), the longitudinal piezoelectric response of domain engineered relaxor-PT crystals is proportional to the product of the longitudinal dielectric permittivity $\varepsilon_{33}$ and the electrostrictive coefficient $\left(Q_{33}-Q_{31}\right)$. In Eqs. (30)-(32), the coefficient $d_{33}$ was not only attributed to the longitudinal $Q_{33}$ but also to the transverse electrostriction $Q_{31}$, because the polarization direction changed as the applied electric field deviated from the polar direction for domain engineered crystals. Table VI gives the piezoelectric coefficients, dielectric permittivities, and electrostrictive coefficients for the domain-engineered PMN- $x$ PT crystals. ${ }^{103}$ The piezoelectric coefficients for [001] poled rhombohedral and orthorhombic crystals were found to be much higher than those of [111] poled orthorhombic and tetragonal counterparts, though the latter possessed higher dielectric constants. This phenomenon can be explained by the anisotropy of the electrostriction, where the highest electrostrictive coefficients $Q_{33}^{*}$ were observed along the $\langle 100\rangle$ directions in perovskite crystals. Thus, the high longitudinal piezoelectric response in perovskite crystals is expected in the $\langle 100\rangle$ poled domain-engineered rhombohedral or orthorhombic crystals, because both the dielectric permittivity and electrostrictive coefficient are enhanced. On the contrary, for $\langle 111\rangle$ and $\langle 110\rangle$ poled domain-engineered tetragonal crystals, the dielectric permittivity is enhanced, but the electrostrictive coefficient greatly decreased. As a result, the piezoelectric coefficient in $\langle 111\rangle$ poled tetragonal crystals doesn't benefit significantly from the engineered domain configuration, since $d_{33}^{*} / d_{33}$ is much smaller in comparison with their rhombohedral and orthorhombic counterparts, as listed in Table VI.

\section{Can piezoelectric activity be improved with electrostriction?}

Ferroelectric materials with a perovskite structure, including BT, PZT ceramics, and PMN-PT, PZN-PT, PIN-PMN-PT single crystals, have been extensively studied as they have a high piezoelectricity. ${ }^{20,104}$ At the present time, efforts in increasing the intrinsic piezoelectric activity of ferroelectrics are mainly focused on the dielectric response. To improve the dielectric response, ferroelectric phase transitions (MPB and PPT) ${ }^{105-108}$ and donor dopants are two generally accepted approaches for use in ferroelectric crystals/ceramics. ${ }^{109,110}$ Furthermore, an electron-irradiated method was applied to the poly(vinylidene fluoride-trifluoroethylene) copolymer P(VDFTrFE) to enhance the dielectric property. ${ }^{8}$ Development over the last 60 years has shown that there is limited room left to further enhance the piezoelectric response in ferroelectrics using these approaches. Meanwhile, it should be noted that the piezoelectricity was enhanced at the cost of the piezoelectric stabilities by these approaches, including the thermal and field (electric field and stress) stabilities. ${ }^{103}$ Section IV B discussed the figure of merit used to evaluate the piezoelectric coefficient $d$ for perovskites, which is the product of the dielectric constant and the electrostrictive coefficient, leading to the conclusion that the piezoelectric response could be improved by enlarging the electrostrictive effect. The importance of this approach has not attracted its deserved attention. One reason is that tuning the electrostrictive coefficients $Q$ is difficult when compared with the dielectric constant, because $Q$ is not sensitive to the phase transformation (PPT or MPB). Second, the electrostrictive coefficient $Q$ increased at the cost of decreased dielectric constant (Eq. (22)), where $\log (Q)$ and $\log \left(\varepsilon / \varepsilon_{0}\right)$ followed a near-linear relationship, as discussed in Sec. IV B. Figure 26 plots the $Q_{33}^{*}$ as a function of $\varepsilon_{33}^{*} / \varepsilon_{0}$ and $Q_{33}^{*}\left(\varepsilon_{33}^{*} / \varepsilon_{0}\right)$ for the domain-engineered PMN-xPT crystals, based on the data listed in Table VI, where the factor $Q_{33}^{*}\left(\varepsilon_{33}^{*} / \varepsilon_{0}\right)$ is proportional to the longitudinal piezoelectric

TABLE VI. Electromechanical properties of domain engineered PMN- $x$ PT crystals. $d_{33}$ is the piezoelectric coefficient of single domain crystal along its polar direction. $d_{33}^{*}$ is the longitudinal piezoelectric coefficient of domain engineered crystals (along their respective nonpolar directions). Data are from Refs. 103 and this work.

\begin{tabular}{llccccc}
\hline \hline Crystals & & Poling & & & & \\
& Phase & direction & $\varepsilon_{33}^{*} / \varepsilon_{0}$ & $d_{33}^{*} \mathrm{pC} / \mathrm{N}$ & $Q_{33}^{*} \mathrm{~m}^{4} / \mathrm{C}^{2}$ & $Q_{31}^{*} \mathrm{~m}^{4} / \mathrm{C}^{2}$ \\
\hline PMN-0.28PT & Rhombohedral & {$[001]$} & 5200 & 1700 & 0.055 & -0.024 \\
PMN-0.32PT & Orthorhombic & {$[001]$} & 5500 & 1800 & 0.056 & -0.022 \\
PMN-0.32PT & Orthorhombic & {$[111]$} & 9000 & 700 & 0.014 & -0.004 \\
PMN-0.37PT & Tetragonal & {$[111]$} & 10000 & 900 & 0.015 & -0.004 \\
\hline \hline
\end{tabular}




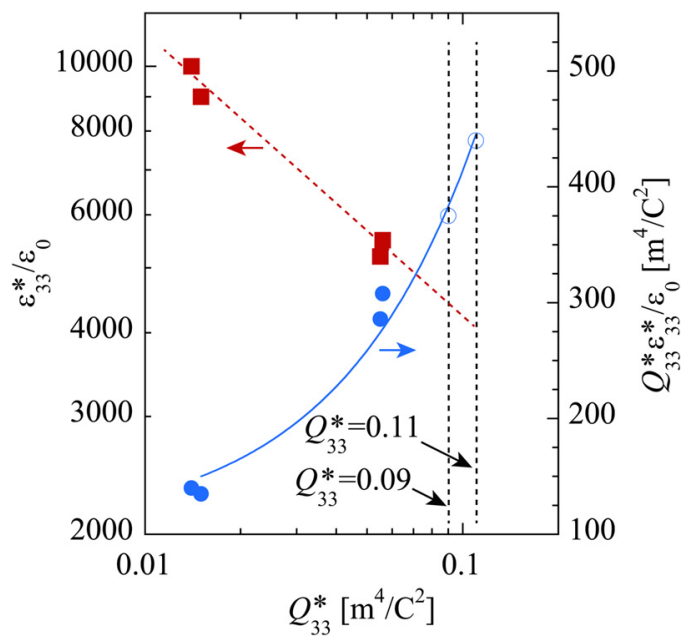

FIG. 26. Dielectric constant $\varepsilon_{33}^{*} / \varepsilon_{0}$ and $Q_{33}^{*} \varepsilon_{33}^{*} / \varepsilon_{0}$ as a function of electrostrictive coefficient $Q_{33}^{*}$ for domain engineered PMN-xPT crystals. Solid circles indicate the experimental data listed in Table VI, while the hollow circles are predicted data. By linear fitting of $\log \left(Q_{33}^{*}\right)$ vs. $\log \left(\varepsilon_{33}^{*} / \varepsilon_{0}\right)$, the dielectric constants $\varepsilon_{33}^{*} / \varepsilon_{0}$ and $Q_{33}^{*} \varepsilon_{33}^{*} / \varepsilon_{0}$ for PMN-PT crystal system were predicted with coefficient $Q_{33}^{*}$ being 0.09 (typical value of PT crystal) and $0.11 \mathrm{~m}^{4} / \mathrm{C}^{2}$ (typical value of BT crystal), respectively.

coefficient. It was observed that $Q_{33}^{*}\left(\varepsilon_{33}^{*} / \varepsilon_{0}\right)$ (the piezoelectric response) could be enhanced by increasing the electrostrictive coefficient $Q_{33}^{*}$, even the dielectric constant showed a contrary trend. From Fig. 26, the piezoelectric response of PMN-PT crystals was expected to increase by $40 \%-60 \%$, with the electrostrictive coefficient approaching the values of the classical ferroelectric perovskite crystals, such as $\mathrm{BaTiO}_{3}$ and PT crystals. The above discussion suggests that the improved piezoelectric activity in domain-engineered relaxor-PT crystals can be realized by enhancing the electrostrictive coefficient. A possible approach to increase the electrostrictive effect in relaxor-PT crystals is to control the degree of ordering for the $\mathrm{A} / \mathrm{B}$ cations, which can be tuned by varying the charge, mass, and size of $\mathrm{A} / \mathrm{B}$ site cations. In addition, a thermal annealing process has been reported to benefit the ordering degree in perovskite systems. ${ }^{111,112}$ Of particular significance is that the enhancement of the electrostrictive coefficients does not sacrifice the piezoelectric stability (including thermal and field stability), as the high piezoelectric activity is not induced by the MPB or PPT, which is promising from the application viewpoint.

In this section, improvement in the piezoelectric activity was discussed from the intrinsic aspect. It should be noted that the piezoelectricity of ferroelectrics could be improved from the extrinsic contribution (domain wall motion), which accounts for up to $50 \%$ of the piezoelectricity in ferroelectric ceramics. However, the intrinsic contribution, which essentially enhances the piezoelectric activity from one material system to another, is the dominant factor in improving the piezoelectric activity. For example, in perovskite-structured ferroelectrics, the intrinsic contribution exhibits a drastic increase from $\mathrm{BaTiO}_{3}(100 \mathrm{pC} / \mathrm{N})$ to PMN-PT $(2000 \mathrm{pC} / \mathrm{N})$ via PZT $(500 \mathrm{pC} / \mathrm{N})$. In contrast to the domain wall motion, enhancement in the intrinsic piezoelectricity does not lead to hysteresis in the strain vs electric-field response. ${ }^{108}$

\section{CONCLUSIONS AND FUTURE PERSPECTIVES}

In this review, the measurement techniques and the origins of electrostriction in ionic crystals were surveyed. The relationship between electrostriction and crystal structure, and the empirical rules for the electrostrictive coefficient $Q$ versus thermal expansion, dielectric constant, and elastic constant were discussed, with emphasis on the perovskite ferroelectrics, because of their high-electrostriction-induced piezoelectric activity.

Electrostriction plays a critical role in the electromechanical properties of ferroelectrics and is worthy of further investigation. Therefore, tuning the electrostrictive coefficient is of particular importance for exploring nextgeneration high-performance electromechanical materials. In the following, some interesting research topics related to the electrostriction in perovskite materials are proposed: (1) Establish a model to accurately describe electrostriction in ionic crystals, incorporating the anisotropy, defects, ordering degree, and other microscopic characteristics. (2) Explore the maximum value of the electrostrictive coefficient $Q$ for perovskite crystals and other ferroelectric systems. To solve this issue, theoretical calculations such as the first-principle method, which provides consistent data for BT and PT crystals, can be employed. (3) Analyze the effects of the A-site cations on electrostriction for perovskite crystals, from the viewpoint of the order-disorder structures. (4) Investigate the relationship between the electrostriction and domain size/domain wall density for ferroelectrics.

\section{ACKNOWLEDGMENTS}

The authors from XJTU acknowledge the supports by the National Nature Science Foundation of China (Grant Nos. 51102193, 51202183, and 51372196), the China Postdoctoral Science Foundation, and the Fundamental Research Funds for the Central Universities.

\section{APPENDIX: THE CALCULATION OF ELECTROSTRICTIVE COEFFICIENT BASED ON PIEZOELECTRIC COEFFICIENT}

The coordinate systems for perovskite-ferroelectric crystals with rhombohedral, orthorhombic, and tetragonal structures and the determination process of the electrostrictive coefficients via piezoelectric response are presented.

For $3 m$ symmetry, the three principal axes (X, Y, and Z)

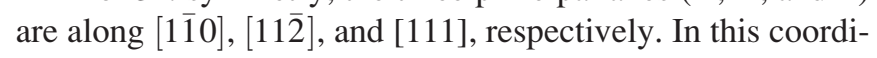
nate system, the electrostrictive coefficients are noted as $Q_{i j k l}^{\mathrm{R}}$. The detailed relations between piezoelectric coefficients and electrostrictive coefficients can be written as follows:

$$
\begin{aligned}
& d_{333}^{\mathrm{R}}=2 P_{3}^{\mathrm{R}} \varepsilon_{33}^{\mathrm{R}} Q_{3333}^{\mathrm{R}}, \\
& d_{311}^{\mathrm{R}}=2 P_{3}^{\mathrm{R}} \varepsilon_{33}^{\mathrm{R}} Q_{3311}^{\mathrm{R}}, \\
& d_{113}^{\mathrm{R}}=2 P_{3}^{\mathrm{R}} \varepsilon_{11}^{\mathrm{R}} Q_{1313}^{\mathrm{R}} .
\end{aligned}
$$

Based on Eqs. (A1)-(A3), the electrostrictive coefficients $Q_{3333}^{\mathrm{R}}, Q_{3311}^{\mathrm{R}}$, and $Q_{1313}^{\mathrm{R}}$ can be determined. 
To compare the electrostrictive properties of crystals with various ferroelectric phases, the values of electrostrictive coefficients are generally transformed to the principal axes of the parent cubic phase of perovskite crystal. The X, $\mathrm{Y}$, and $\mathrm{Z}$ axes of cubic phase are along [100], [010], and [001] directions, respectively. In this coordinate system, there are three independent electrostrictive coefficients: $Q_{11}^{\mathrm{C}}, Q_{12}^{\mathrm{C}}$, and $Q_{44}^{\mathrm{C}}$, where $Q_{11}^{\mathrm{C}}=Q_{111}^{\mathrm{C}}, Q_{12}^{\mathrm{C}}=Q_{1122}^{\mathrm{C}}$, and $Q_{44}^{\mathrm{C}}=2 Q_{1212}^{\mathrm{C}}$ (note: some references use the equation $Q_{44}^{\mathrm{C}}=4 Q_{1212}^{\mathrm{C}}{ }^{113}$ ). By axis transformation, ${ }^{1}$ the $Q_{i j k l}^{\mathrm{R}}$ can be expressed as

$$
\begin{gathered}
Q_{3333}^{\mathrm{R}}=\frac{1}{3}\left(Q_{11}^{\mathrm{C}}+2 Q_{12}^{\mathrm{C}}+2 Q_{44}^{\mathrm{C}}\right), \\
Q_{3311}^{\mathrm{R}}=\frac{1}{3}\left(Q_{11}^{\mathrm{C}}+2 Q_{12}^{\mathrm{C}}-2 Q_{44}^{\mathrm{C}}\right), \\
Q_{1313}^{\mathrm{R}}=Q_{2323}^{\mathrm{R}}=\frac{1}{3}\left(Q_{11}^{\mathrm{C}}-Q_{12}^{\mathrm{C}}+\frac{1}{2} Q_{44}^{\mathrm{C}}\right) .
\end{gathered}
$$

Thus, the electrostrictive coefficients $Q_{11}^{\mathrm{C}}, Q_{12}^{\mathrm{C}}$, and $Q_{44}^{\mathrm{C}}$ can be calculated based on Eqs. (A4)-(A6).

For $m m 2$ symmetry, the three principal axes (X, Y, and Z) are along $[011]$, [100], and [011], respectively. In this coordinate system, the electrostrictive coefficients are noted as $Q_{i j k l}^{\mathrm{O}}$. The detailed relations between piezoelectric coefficients and electrostrictive coefficients can be written as follows:

$$
\begin{aligned}
& d_{333}^{\mathrm{O}}=2 P_{3}^{\mathrm{O}} \varepsilon_{33}^{\mathrm{O}} Q_{3333}^{\mathrm{O}}, \\
& d_{113}^{\mathrm{O}}=2 P_{3}^{\mathrm{O}} \varepsilon_{11}^{\mathrm{O}} Q_{1313}^{\mathrm{O}}, \\
& d_{223}^{\mathrm{O}}=2 P_{3}^{\mathrm{O}} \varepsilon_{22}^{\mathrm{O}} Q_{2323}^{\mathrm{O}} .
\end{aligned}
$$

The relationship between the electrostrictive coefficients $Q_{i j k l}^{\mathrm{O}}$ and $Q_{i j k l}^{\mathrm{C}}$ can be expressed in Eqs. (A10)-(A12)

$$
\begin{gathered}
Q_{3333}^{\mathrm{O}}=\frac{1}{2}\left(Q_{11}^{\mathrm{C}}+Q_{12}^{\mathrm{C}}+Q_{44}^{\mathrm{C}}\right), \\
Q_{1313}^{\mathrm{O}}=\frac{1}{2}\left(Q_{11}^{\mathrm{C}}-Q_{12}^{\mathrm{C}}\right), \\
Q_{2323}^{\mathrm{O}}=\frac{1}{2} Q_{44}^{\mathrm{C}} .
\end{gathered}
$$

For $4 \mathrm{~mm}$ symmetry, the three principal axes (X, Y, and Z) are along [100], [010], and [001], respectively, being same to the principal axes of cubic phase. Thus, the electrostrictive coefficients can be directly noted as $Q_{i j k l}^{\mathrm{C}}$. The detailed relations between piezoelectric coefficients and electrostrictive coefficients can be written as follows:

$$
\begin{aligned}
& d_{333}^{\mathrm{T}}=2 P_{3}^{\mathrm{T}} \varepsilon_{33}^{\mathrm{T}} Q_{3333}^{\mathrm{T}}, \\
& d_{311}^{\mathrm{T}}=2 P_{3}^{\mathrm{T}} \varepsilon_{33}^{\mathrm{T}} Q_{3311}^{\mathrm{T}}, \\
& d_{113}^{\mathrm{T}}=2 P_{3}^{\mathrm{T}} \varepsilon_{11}^{\mathrm{T}} Q_{1313}^{\mathrm{T}} .
\end{aligned}
$$

${ }^{1}$ R. E. Newnham, Properties of Materials: Anisotropy, Symmetry, Structure (Oxford, New York, 2005).

${ }^{2}$ K. Uchino, S. Nomura, L. E. Cross, S. J. Jang, and R. E. Newnham, "Electrostrictive effect in lead magnesium niobate single crystals," J. Appl. Phys. 51, 1142 (1980).

${ }^{3}$ K. Uchino, S. Nomura, L. E. Cross, R. E. Newnham, and S. J. Jang, "Electrostrictive effect in perovskites and its transducer applications," J. Mater. Sci. 16, 569 (1981).

${ }^{4}$ L. E. Cross, S. J. Jang, R. E. Newnham, S. Nomura, and K. Uchino, "Large electrostrictive effects in relaxor ferroelectrics," Ferroelectrics 23, 187 (1980).

${ }^{5}$ S. Nomura, J. Kuwata, S. J. Jang, L. E. Cross, and R. E. Newnham, "Electrostriction in $\mathrm{Pb}\left(\mathrm{Zn}_{1 / 3} \mathrm{Nb}_{2 / 3}\right) \mathrm{O}_{3}$," Mater. Res. Bull. 14, 769 (1979).

${ }^{6}$ S. J. Jang, K. Uchino, S. Nomura, and L. E. Cross, "Electrostrictive behavior of lead magnesium niobate based ceramic dielectrics," Ferroelectrics 27, 31 (1980).

${ }^{7}$ K. Uchino, L. E. Cross, R. E. Newnham, and S. Nomura, "Electrostrictive effects in non-polar perovskites," Phase Transitions 1, 333 (1980).

${ }^{8} \mathrm{Q}$. M. Zhang, V. Bharti, and X. Zhao, "Giant electrostriction and relaxor ferroelectric behavior in electron-irradiated poly(vinylidene fluoride-trifluoroethylene) copolymer," Science 280, 2101 (1998).

${ }^{9}$ Q. M. Zhang, H. Li, M. Poh, F. Xia, Z. Y. Cheng, H. Xu, and C. Huang, "An all-organic composite actuator material with a high dielectric constant," Nature 419, 284 (2002).

${ }^{10}$ Q. M. Zhang, J. Su, C. H. Kim, R. Ting, and R. Capps, “An experimental investigation of electromechanical responses in a polyurethane elastomer," J. Appl. Phys. 81, 2770 (1997)

${ }^{11}$ R. E. Newnham, V. Sundar, R. Yimnirun, J. Su, and Q. M. Zhang, "Electrostriction: Nonlinear electromechanical coupling in solid dielectrics," J. Phys. Chem. B 101, 10141 (1997).

${ }^{12}$ W. Lehmann, H. Skupin, C. Tolksdorf, E. Gebhard, R. Zentel, P. Krüger, M. Lösche, and F. Kremer, "Giant lateral electrostriction in ferroelectric liquid-crystalline elastomers," Nature 410, 447 (2001).

${ }^{13}$ R. Pelrine, R. Kornbluh, and G. Kofod, "High-strain actuator materials based on dielectric elastomers," Adv. Mater. 12, 1223 (2000).

${ }^{14}$ R. Pelrine, R. Kornbluh, J. Joseph, R. Heydt, Q. Pei, and S. Chiba, "Highfield deformation of elastomeric dielectrics for actuators," Mater. Sci. Eng.: C 11, 89 (2000).

${ }^{15}$ M. Zhenyi, J. I. Scheinbeim, J. W. Lee, and B. A. Newman, "High field electrostrictive response of polymers," J. Polym. Sci. B 32, 2721 (1994).

${ }^{16} \mathrm{H}$. Jaffe and D. A. Berlincourt, "Piezoelectric transducer materials," Proc. IRE 53, 1372 (1965).

${ }^{17}$ B. Jaffe, W. R. Cook, Jr, and H. Jaffe, Piezoelectric Ceramics (Academic, New York, 1971).

${ }^{18}$ S. E. Park and T. R. Shrout, "Ultrahigh strain and piezoelectric behavior in relaxor based ferroelectric single crystals," J. Appl. Phys. 82, 1804 (1997).

${ }^{19}$ S. E. Park and T. R. Shrout, "Relaxor based ferroelectric single crystals for electro-mechanical actuators," Mater. Res. Innovations 1, 20 (1997).

${ }^{20} \mathrm{~S}$. Zhang and F. Li, "High performance ferroelectric relaxor- $\mathrm{PbTiO}_{3}$ single crystals: Status and perspective,” J. Appl. Phys. 111, 031301 (2012).

${ }^{21} \mathrm{~F}$. Li, S. Zhang, Z. Xu, X. Wei, and T. R. Shrout, "Critical property in relaxor- $\mathrm{PbTiO}_{3}$ single crystals-Shear piezoelectric response," Adv. Funct. Mater. 21, 2118 (2011).

${ }^{22} \mathrm{X}$. Li and H. Luo, "The growth and properties of relaxor-based ferroelectric single crystals," J. Am. Ceram. Soc. 93, 2915 (2010).

${ }^{23}$ N. Luo, Y. Li, Z. Xia, and Q. Li, "Progress in lead-based ferroelectric and antiferroelectric single crystals: Composition modification, crystal growth and properties," CrystEngComm 14, 4547 (2012).

${ }^{24}$ E. Fukada, "History and recent progress in piezoelectric polymers," IEEE Trans. Ultrason. Ferroelectr. Freq. Control 47, 1277 (2000).

${ }^{25} \mathrm{~T}$. Furukawa and N. Seo, "Electrostriction as the origin of piezoelectricity in ferroelectric polymers," Jpn. J. Appl. Phys., Part 1 29, 675 (1990).

${ }^{26} \mathrm{~F}$. Li, L. Jin, Z. Xu, D. Wang, and S. Zhang, "Electrostrictive effect in $\mathrm{Pb}\left(\mathrm{Mg}_{1 / 3} \mathrm{Nb}_{2 / 3}\right) \mathrm{O}_{3}-x \mathrm{PbTiO}_{3}$ crystals," Appl. Phys. Lett. 102, 152910 (2013).

${ }^{27}$ G. Viola, T. Saunders, X. Wei, K. B. Chong, H. Luo, M. J. Reece, and H. Yan, "Contribution of piezoelectric effect, electrostriction and ferroelectric/ferroelastic switching to strain-electric field response of dielectrics," J. Adv. Dielectr. 3, 1350007 (2013).

${ }^{28}$ L. E. Cross, "Relaxor ferroelectrics," Ferroelectrics 76, 241 (1987).

${ }^{29}$ Z. G. Ye, "Relaxor ferroelectric complex perovskites: Structure, properties and phase transitions," Key Eng. Mater. 155-156, 81 (1998). 
${ }^{30}$ Z. Kighelman, D. Damjanovic and N. Setter, "Dielectric and electromechanical properties of ferroelectric-relaxor $0.9 \mathrm{~Pb}\left(\mathrm{Mg}_{1 / 3} \mathrm{Nb}_{2 / 3}\right) \mathrm{O}_{3}-0.1 \mathrm{PbTiO}_{3}$ thin film," J. Appl. Phys. 90, 4682 (2001).

${ }^{31}$ J. F. Nye, Physical Properties of Crystals: Their Representation by Tensors and Matrices (Oxford, New York, 1957).

${ }^{32}$ M. E. Lines and A. M. Glass, Principles and Applications of Ferroelectrics and Related Materials (Oxford, New York, 1979).

${ }^{33}$ K. Rittenmyer, A. S. Bhalla, and L. E. Cross, "Temperature dependence of the dielectric constant of $\mathrm{KMnF}_{3}$," Ferroelectr., Lett. Sect. 9, 161 (1989).

${ }^{34}$ F. Li, S. Zhang, Z. Xu, D. Lin, J. Gao, Z. Li, and L. Wang, "An efficient way to enhance output strain for shear mode $\mathrm{Pb}\left(\mathrm{In}_{1 / 2} \mathrm{Nb}_{1 / 2}\right) \mathrm{O}_{3}-\mathrm{Pb}\left(\mathrm{Mg}_{1 / 3} \mathrm{Nb}_{2 / 3}\right) \mathrm{O}_{3}-\mathrm{PbTiO}_{3}$ crystals: Applying uniaxial stress perpendicular to polar direction," Appl. Phys. Lett. 100, 192901 (2012).

${ }^{35}$ J. Gao, Z. Xu, F. Li, C. Zhang, Z. Li, X. Wu, L. Wang, Y. Liu, G. Liu, and $\mathrm{H}$. He, "Pyroelectric properties of rhombohedral and tetragonal $\mathrm{Pb}\left(\mathrm{In}_{1 / 2} \mathrm{Nb}_{1 / 2}\right) \mathrm{O}_{3}-\mathrm{Pb}\left(\mathrm{Mg}_{1 / 3} \mathrm{Nb}_{2 / 3}\right) \mathrm{O}_{3}-\mathrm{PbTiO}_{3}$ crystals," J. Appl. Phys. 110, 106101 (2011).

${ }^{36}$ ANSI/IEEE Standard No. 176-1987, IEEE Standard on Piezoelectricity, IEEE, New York, 1987.

${ }^{37}$ D. Berlincourt and H. Jaffe, "Elastic and piezoelectric coefficients of single-crystal barium titanate," Phys. Rev. 111, 143 (1958).

${ }^{38}$ S. T. Misture, S. M. Pilgrim, J. C. Hicks, C. T. Blue, E. A. Payzant, and C. R. Hubbard, "Measurement of the electrostrictive coefficients of modified lead magnesium niobate using neutron powder diffraction," Appl. Phys. Lett. 72, 1042 (1998).

${ }^{39}$ C. T. Blue, J. C. Hicks, and S. R. Winzer, "Investigation of crystallographic and bulk strain in doped lead magnesium niobate," J. Appl. Phys. 82, 3972 (1997).

${ }^{40} \mathrm{G}$. Zorn, W. Wersing, and H. Göbel, "Comparison of piezoelectric constants of PZT ceramics with values calculated from electrostrictive coefficients," Jpn. J. Appl. Phys., Part 1 24-2(Suppl.), 724 (1985).

${ }^{41}$ J. Zhao, A. E. Glazounov, Q. M. Zhang, and B. Toby, "Neutron diffraction study of electrostrictive coefficients of prototype cubic phase of relaxor ferroelectric $\mathrm{PbMg}_{1 / 3} \mathrm{Nb}_{2 / 3} \mathrm{O}_{3}$," Appl. Phys. Lett. 72, 1048 (1998).

${ }^{42}$ J. Toulouse and R. K. Pattnaik, "Nonlinear electrostriction in the mixed ferroelectric $\mathrm{KTa}_{1-x} \mathrm{Nb}_{x} \mathrm{O}_{3}$," Phys. Rev. B 65, 024107 (2001).

${ }^{43} \mathrm{~F}$. Craciun, "Strong variation of electrostrictive coupling near an intermediate temperature of relaxor ferroelectrics," Phys. Rev. B 81, 184111 (2010).

${ }^{44}$ L. Liang, Y. L. Li, L. Q. Chen, S. Y. Hu, and G. H. Lu, "A thermodynamic free energy function for potassium niobate," Appl. Phys. Lett. 94, 072904 (2009).

${ }^{45}$ J. J. Wang, F. Y. Meng, X. Q. Ma, M. X. Xu, and L. Q. Chen, "Lattice, elastic, polarization, and electrostrictive properties of $\mathrm{BaTiO}_{3}$ from firstprinciples," J. Appl. Phys. 108, 034107 (2010).

${ }^{46}$ N. W. Ashcroft and N. D. Mermin, Solid State Physics (Brooks Cole, Philadelphia, 1976).

${ }^{47}$ C. Kittel, Introduction to Solid State Physics, 8th ed. (Wiley, New York, 2004).

${ }^{48}$ Comments on the negative coefficient $Q_{11}$. Negative $Q_{11}$ was reported in fluorite crystals, as listed in Table II. The possible reasons of negative $Q_{11}$ may relate to two aspects. First, there are some mistakes in determination of $Q_{11}$ because the electric-field induced strain is quite small for fluorite crystals (low dielectric constant). Second, although the Eq. (18) can explain the positive coefficient $Q_{11}$ for ionic crystals, in some cases the model for Eq. (18) may deviate from the real condition. Eq. (18) comes from the simplest rigid ion model, where only the interaction among nearest ions is considered and the crystal structure isn't taken into account.

${ }^{49}$ K. Uchino, S. Nomura, K. Vedam, R. E. Newnham, and L. E. Cross, "Pressure dependence of the refractive index and dielectric constant in a fluoroperovskite, $\mathrm{KMgF}_{3}$," Phys. Rev. B 29, 6921 (1984).

${ }^{50} \mathrm{R}$. A. Anderson, "Mechanical stress in a dielectric solid from a uniform electric field," Phys. Rev. B 33, 1302 (1986).

${ }^{51}$ M. J. Haun, E. S. Furman, J. Jang, and L. E. Cross, "Thermodynamic theory of the lead zirconate-titanate solid solution system, part I-part V: Phenomenology," Ferroelectrics 99, 13 (1989).

${ }^{52} \mathrm{~A}$. W. Warner, M. Onoe, and G. A. Coquin, "Determination of elastic and piezoelectric constants for crystals in class ( $3 m)$," J. Acoust. Soc. Am. 42, 1223 (1967).

${ }^{53} \mathrm{~T}$. Yamada, "Electromechanical properties of oxygen-octahedra ferroelectric crystals," J. Appl. Phys. 43, 328 (1972).

${ }^{54}$ V. Sundar, J. F. Li, D. Viehland, and R. E. Newnham, "Interferometric evaluation of electrostriction coefficients," Mater. Res. Bull. 31, 555 (1996)
${ }^{55}$ K. Rittenmyer, A. S. Bhalla, and L. E. Cross, "Electrostriction in fluoride perovskites," Mater. Lett. 7, 380 (1989).

${ }^{56} \mathrm{~V}$. Sundar and R. E. Newnham, "Electrostriction and polarization," Ferroelectrics 135, 431 (1992).

${ }^{57} \mathrm{~V}$. Sundar and R. E. Newnham, "Converse method measurements of electrostriction coefficients in low-K dielectrics," Mater. Res. Bull. 31, 545 (1996).

${ }^{58}$ R. Srinivasan and K. Srinivasan, "Strain dependence of static and high frequency dielectric constants of some alkali halides," J. Phys. Chem. Solids 33, 1079 (1972).

${ }^{59}$ A. D. B. Woods, W. Cochran, and B. N. Brockhouse, "Lattice dynamics of alkali halide crystals," Phys. Rev. 119, 980 (1960).

${ }^{60}$ Q. M. Zhang, J. Zhao, T. Shrout, N. Kim, L. E. Cross, A. Amin, and B. M. Kulwicki, "Characteristics of the electromechanical response and polarization of electric field biased ferroelectrics," J. Appl. Phys. 77, 2549 (1995).

${ }^{61}$ V. S. Vikhnin, R. Blinc, and R. Pirc, "Mechanisms of electrostriction and giant piezoelectric effect in relaxor ferroelectrics," J. Appl. Phys. 93, 9947 (2003).

${ }^{62}$ R. Pirc, R. Blinc and V. S. Vikhnin, "Effect of polar nanoregions on giant electrostriction and piezoelectricity in relaxor ferroelectrics," Phys. Rev. B 69, 212105 (2004).

${ }^{63}$ G. A. Samara, "Pressure and temperature dependences of the dielectric properties of the perovskites $\mathrm{BaTiO}_{3}$ and $\mathrm{SrTiO}_{3}$," Phys. Rev. 151, 378 (1966).

${ }^{64}$ H. Uwe, H. Unoki, Y. Fujii, and T. Sakudo, "Stress induced ferroelectricity in $\mathrm{KTaO}_{3}$," Solid State Commun. 13, 737 (1973).

${ }^{65} \mathrm{~N}$. Setter and L. E. Cross, "An optical study of the ferroelectric relaxors $\mathrm{Pb}\left(\mathrm{Mg}_{1 / 3} \mathrm{Nb}_{2 / 3}\right) \mathrm{O}_{3}, \quad \mathrm{~Pb}\left(\mathrm{Sc}_{1 / 2} \mathrm{Ta}_{1 / 2}\right) \mathrm{O}_{3}, \quad$ and $\mathrm{Pb}\left(\mathrm{Sc}_{1 / 2} \mathrm{Nb}_{1 / 2}\right) \mathrm{O}_{3}$," Ferroelectrics 37, 551 (1981).

${ }^{66}$ J. Kuwata, K. Uchino, and S. Nomura, "Diffuse phase transition in lead zinc niobate," Ferroelectrics 22, 863 (1978).

${ }^{67}$ K. Uchino, L. E. Cross, R. E. Newnham, and S. Nomura, "Electrostrictive effects in antiferroelectric perovskites," J. Appl. Phys. 52, 1455 (1981)

${ }^{68}$ S. Nomura, S. J. Jang, L. E. Cross, and R. E. Newnham, "Structure and dielectric properties of materials in the solid solution system $\mathrm{Pb}\left(\mathrm{Mg}_{1 / 3} \mathrm{Nb}_{2 / 3}\right) \mathrm{O}_{3}: \mathrm{Pb}\left(\mathrm{W}_{1 / 2} \mathrm{Mg}_{1 / 2}\right) \mathrm{O}_{3}$," J. Am. Ceram. Soc. 62, 485 (1979).

${ }^{69}$ D. Damjanovic, "Stress and frequency dependence of the direct piezoelectric effect in ferroelectric ceramics," J. Appl. Phys. 82, 1788 (1997).

${ }^{70} \mathrm{D}$. Damjanovic, "Ferroelectric, dielectric and piezoelectric properties of ferroelectric thin films and ceramics," Rep. Prog. Phys. 61, 1267 (1998).

${ }^{71}$ V. Porokhonskyy, L. Jin, and D. Damjanovic, "Separation of piezoelectric grain resonance and domain wall dispersion in $\mathrm{Pb}(\mathrm{Zr}, \mathrm{Ti}) \mathrm{O}_{3}$ ceramics," Appl. Phys. Lett. 94, 212906 (2009).

${ }^{72}$ L. Jin, Z. He, and D. Damjanovic, "Nanodomains in $\mathrm{Fe}^{+3}$-doped lead zirconate titanate ceramics at the morphotropic phase boundary do not correlate with high properties," Appl. Phys. Lett. 95, 012905 (2009).

${ }^{73}$ L. Jin, V. Porokhonskyy, and D. Damjanovic, "Domain wall contributions in $\mathrm{Pb}(\mathrm{Zr}, \mathrm{Ti}) \mathrm{O}_{3}$ ceramics at morphotropic phase boundary: A study of dielectric dispersion,” Appl. Phys. Lett. 96, 242902 (2010).

${ }^{74} \mathrm{O}$. Noblanc and P. Gaucher, "Influence of domain walls on piezoelectric and electrostrictive properties of PMN-PT (65/35) ceramics," Ferroelectrics 160, 145 (1994).

${ }^{75}$ P. M. Weaver, M. G. Cain, and M. Stewart, "Temperature dependence of strain-polarization coupling in ferroelectric ceramics," Appl. Phys. Lett. 96, 142905 (2010)

${ }^{76}$ D. H. Kang, Y. H. Lee, and K. H. Yoon, "Phase transition, dielectric and electrostrictive behaviors in $(1-x) \mathrm{PYN}-x \mathrm{PMN}$," J. Mater. Res. 13, 984 (1998).

${ }^{77}$ G. Haertling, "PLZT electrooptic materials and applications-a review," Ferroelectrics 75, 25 (1987).

${ }^{78}$ S. A. Sheets, A. N. Soukhojak, N. Ohashi, and Y. M. Chiang, "Relaxor single crystals in the $\left(\mathrm{Bi}_{1 / 2} \mathrm{Na}_{1 / 2}\right)_{1-x} \mathrm{Ba}_{x} \mathrm{Zr}_{y} \mathrm{Ti}_{1-y} \mathrm{O}_{3}$ system exhibiting high electrostrictive strain," J. Appl. Phys. 90, 5287 (2001).

${ }^{79}$ V. Bobnar, B. Malič, J. Holc, M. Kosec, R. Steinhausen, and H. Beige, "Electrostrictive effect in lead-free relaxor $\mathrm{K}_{0.5} \mathrm{Na}_{0.5} \mathrm{NbO}_{3}-\mathrm{SrTiO}_{3}$ ceramic system," J. Appl. Phys. 98, 024113 (2005).

${ }^{80} \mathrm{~J}$. Hao, W. Bai, W. Li, B. Shen, and J. Zhai, "Phase transitions, relaxor behavior, and electrical properties in $(1-x)\left(\mathrm{Bi}_{0.5} \mathrm{Na}_{0.5}\right) \mathrm{TiO}_{3}-x\left(\mathrm{~K}_{0.5} \mathrm{Na}_{0.5}\right) \mathrm{NbO}_{3} \quad$ lead-free piezoceramics," J. Mater. Res. 27, 2943 (2012). 
${ }^{81}$ S. T. Zhang, F. Yan, B. Yang, and W. Cao, "Phase diagram and electrostrictive properties of $\mathrm{Bi}_{0.5} \mathrm{Na}_{0.5} \mathrm{TiO}_{3}-\mathrm{BaTiO}_{3}-\mathrm{K}_{0.5} \mathrm{Na}_{0.5} \mathrm{NbO}_{3}$ ceramics," Appl. Phys. Lett. 97, 122901 (2010).

${ }^{82}$ S. T. Zhang, A. B. Kounga, W. Jo, C. Jamin, K. Seifert, T. Granzow, J. Rödel, and D. Damjanovic, "High-strain lead-free antiferroelectric electrostrictors," Adv. Mater. 21, 4716 (2009).

${ }^{83}$ H. S. Han, W. Jo, J. K. Kang, C. W. Ahn III, W. Kim, K. K. Ahn, and J. $\mathrm{S}$. Lee, "Incipient piezoelectrics and electrostriction behavior in Sn-doped $\mathrm{Bi}_{1 / 2}\left(\mathrm{Na}_{0.82} \mathrm{~K}_{0.18}\right)_{1 / 2} \mathrm{TiO}_{3}$ lead-free ceramics," J. Appl. Phys. 113, 154102 (2013).

${ }^{84}$ S. G. Lee, R. G. Monteiro, R. S. Feigelson, H. S. Lee, M. Lee, and S. E. Park, "Growth and electrostrictive properties of $\mathrm{Pb}\left(\mathrm{Mg}_{1 / 3} \mathrm{Nb}_{2 / 3}\right) \mathrm{O}_{3}$ crystals," Appl. Phys. Lett. 74, 1030 (1999).

${ }^{85}$ A. L. Kholkin, E. K. Akdogan, A. Safari, P. F. Chauvy, and N. Setter, "Characterization of the effective electrostriction coefficients in ferroelectric thin films," J. Appl. Phys. 89, 8066 (2001).

${ }^{86} \mathrm{~A}$. Kvasov and A. K. Tagantsev, "Positive effective $Q_{12}$ electrostrictive coefficient in perovskites,” J. Appl. Phys. 112, 094106 (2012).

${ }^{87}$ P. M. Weaver, M. G. Cain, and M. Stewart, "Temperature dependence of high field electromechanical coupling in ferroelectric ceramics," J. Phys. D: Appl. Phys. 43, 165404 (2010).

${ }^{88}$ X. Li, S. G. Lu, X. Z. Chen, H. Gu, X. S. Qian, and Q. M. Zhang, "Pyroelectric and electrocaloric materials," J. Mater. Chem. C 1, 23 (2013).

${ }^{89}$ I. Jankowska-Sumara, K. Roleder, A. Majchrowski, and J. Zmija, "Nonlinear electrostrictive properties of $\mathrm{PbZrO}_{3}: \mathrm{Sn}$ single crystals with antiferroelectric phase transitions," J. Adv. Dielectr. 1, 223 (2011).

${ }^{90}$ W. Pan, Q. Zhang, A. S. Bhalla, and L. E. Cross, "Field-induced strain in single-crystal BaTiO 3 ," J. Am. Ceram. Soc. 71, C-302 (1988).

${ }^{91}$ G. R. Barsch, B. N. N. Achar, and L. E. Cross, "Phenomenological theory of the temperature variation of electrostriction of ferroelectrics in the paraelectric phase," Ferroelectrics 35, 191 (1981).

${ }^{92}$ For $Q_{i j}^{\mathrm{C}}$ and $Q_{i j}^{\mathrm{R}}$ coefficient, the superscripts $\mathrm{C}$ and $\mathrm{R}$ denote that the electrostrictive coefficients $Q$ are measured in the standard coordinate system of cubic $m \overline{3} m$ and rhombohedral $3 m$ phase of perovskite crystals (see details in the Appendix).

${ }^{93}$ J. Yin, B. Jiang, and W. Cao, "Elastic, piezoelectric, and dielectric properties of $0.955 \mathrm{~Pb}\left(\mathrm{Zn}_{1 / 3} \mathrm{Nb}_{2 / 3}\right) \mathrm{O}_{3}-0.45 \mathrm{PbTiO}_{3}$ single crystal with designed multidomains," IEEE Trans. Ultrason. Ferroelectr. Freq. Control 47, 285 (2000)

${ }^{94}$ R. Zhang, B. Jiang, W. Cao, and A. Amin, "Complete set of material constants of $0.93 \mathrm{~Pb}\left(\mathrm{Zn}_{1 / 3} \mathrm{Nb}_{2 / 3}\right) \mathrm{O}_{3}-0.07 \mathrm{PbTiO}_{3}$ domain engineered single crystal," J. Mater. Sci. Lett. 21, 1877 (2002).

${ }^{95}$ R. Zhang, B. Jiang, W. Jiang, and W. Cao, "Complete set of properties of $0.92 \mathrm{~Pb}\left(\mathrm{Zn}_{1 / 3} \mathrm{Nb}_{2 / 3}\right) \mathrm{O}_{3}-0.08 \mathrm{PbTiO}_{3}$ single crystal with engineered domains," Mater. Lett. 57, 1305 (2003).

${ }^{96}$ S. Zhang, L. Lebrun, C. A. Randall, and T. R. Shrout, “Orientation dependence properties of modified tetragonal $0.88 \mathrm{~Pb}\left(\mathrm{Zn}_{1 / 3} \mathrm{Nb}_{2 / 3}\right) \mathrm{O}_{3}-0.12 \mathrm{PbTiO}_{3}$ single crystals," Phys. Status Solidi A 202, 151 (2005).
${ }^{97}$ H. Cao, V. H. Schmidt, R. Zhang, W. Cao, and H. Luo, "Elastic, piezoelectric, and dielectric properties of $0.58 \mathrm{~Pb}\left(\mathrm{Mg}_{1 / 3} \mathrm{Nb}_{2 / 3}\right) \mathrm{O}_{3}-0.42 \mathrm{PbTiO}_{3}$ single crystal,” J. Appl. Phys. 96, 549 (2004).

${ }^{98} Q_{i j}^{*}$, the superscript * denotes that the electrostrictive coefficients are measured in a new coordinate system (after axis transformation). $Q_{i j}$ denotes that it is measured in standard coordinate. The standard coordinate systems of $3 \mathrm{~m}, 4 \mathrm{~mm}$, $\mathrm{mm} 2$ symmetries are listed in the Appendix.

${ }^{99}$ X. B. Ren, "Large electric-field-induced strain in ferroelectric crystals by pointdefect-mediated reversible domain switching," Nature Mater. 3, 91 (2004).

${ }^{100}$ E. Burcsu, G. Ravichandran, and K. Bhattacharya, "Large strain electrostrictive actuation in barium titanate," Appl. Phys. Lett. 77, 1698 (2000).

${ }^{101}$ E. Burcsu, G. Ravichandran, and K. Bhattacharya, "Large electrostrictive actuation of barium titanate single crystals," J. Mech. Phys. Solids 52, 823 (2004).

${ }^{102}$ D. Damjanovic, "Hysteresis in piezoelectric and ferroelectric materials," in Science of Hysteresis, edited by G. Bertotti and I. Mayergoyz (Elsevier, Amsterdam, 2005), Vol. III, pp. 337-465.

${ }^{103}$ F. Li, S. Zhang, Z. Xu, X. Wei, J. Luo, and T. R. Shrout, "Composition and phase dependence of the intrinsic and extrinsic piezoelectric activity of domain engineered $(1-x) \mathrm{Pb}\left(\mathrm{Mg}_{1 / 3} \mathrm{Nb}_{2 / 3}\right) \mathrm{O}_{3}-x \mathrm{PbTiO}_{3}$ crystals," J. Appl. Phys. 108, $034106(2010)$

${ }^{104}$ F. Li, S. Zhang, Z. Li, and Z. Xu, "Recent development on relaxorPbTiO3 single crystals: The origin of high piezoelectric response," Progress in Physics 32, 178 (2012) (in Chinese).

${ }^{105}$ W. Liu and X. Ren, "Large piezoelectric effect in Pb-free ceramics," Phys. Rev. Lett. 103, 257602 (2009).

${ }^{106}$ D. Damjanovic, "A morphotropic phase boundary system based on polarization rotation and polarization extension,” Appl. Phys. Lett. 97, 062906 (2010).

${ }^{107}$ M. Ahart, M. Somayazulu, R. E. Cohen, P. Ganesh, P. Dera, H. K. Mao, R. J. Hemley, Y. Ren, P. Liermann, and Z. Wu, "Origin of morphotropic phase boundaries in ferroelectrics," Nature 451, 545 (2008).

${ }^{108}$ D. Damjanovic, "Contributions to the piezoelectric effect in ferroelectric single crystals and ceramics," J. Am. Ceram. Soc. 88, 2663 (2005).

${ }^{109}$ L. E. Cross, "Ferroelectric ceramics: Tailoring properties for specific applications," in Ferroelectric Ceramics, edited by N. Setter and E. L. Colla (Birkhäuser, Basel, 1993), pp. 1-85.

${ }^{110}$ L. Eyraud, B. Guiffard, L. Lebrun, and D. Guyomar, "Interpretation of the softening effect in PZT ceramics near the morphotropic phase boundary," Ferroelectrics 330, 51 (2006).

${ }^{111}$ N. Setter and L. E. Cross, "The role of B-site cation disorder in diffuse phase transition behavior of perovskite ferroelectrics," J. Appl. Phys. 51, 4356 (1980).

${ }^{112}$ N. Setter and L. E. Cross, "The contribution of structural disorder to diffuse phase transitions in ferroelectrics," J. Mater. Sci. 15, 2478 (1980).

${ }^{113}$ M. Davis, M. Budimir, D. Damjanovic, and N. Setter, "Rotator and extender ferroelectrics: Importance of the shear coefficient to the piezoelectric properties of domain-engineered crystals and ceramics," J. Appl. Phys. 101, 054112 (2007). 